\title{
ON THE SELF-HOMOTOPY EQUIVALENCES OF THE WEDGE OF CERTAIN COMPLEXES
}

\author{
By KOHHEI YAMAguchi
}

\section{$\S 1$. Introduction.}

The set of homotopy classes of self-homotopy equivalences of a based space $X$, which is denoted by $\mathrm{Eq}(X)$, is a group with the multiplication defined by the composition of maps. This group $\mathrm{Eq}(X)$ has now been studied by many authors since the paper of W.D. Barcus and M.G. Barratt ([2]) appeared in 1958. However, generally speaking, we have not yet obtained an effective method for calculating it except classical ones, and the structure of it also has not been clarified sufficiently.

In this paper, we study the group $\mathrm{Eq}(\mathrm{K})$ for a $C W$-complex $K$ such as

$$
K=\bigvee_{h=1}^{r} K_{h}, K_{h}=S^{n} \bigcup_{\alpha_{h}} e^{n+k+1} \quad(n \geqq 3, k \geqq 1)
$$

under the condition that the attaching class $\alpha_{h} \in \pi_{n+k}\left(S^{n}\right)$ is a double suspension, $\alpha_{h}=E^{2} \alpha_{h}^{\prime \prime}$, and both $\alpha_{h}$ and $\alpha_{h}^{\prime}=E \alpha_{h}^{\prime \prime}$ have the same order $m_{h}$, for $1 \leqq h \leqq r$. However, the case $r=1$ has already been treated by S. Oka in [6], so we will consider the case $r \geqq 2$.

For each based topological space $X$ and $Y$, we denote by $[X, Y]$ the homotopy set of all based maps from $X$ to $Y$, and also by $[\beta, \gamma]$ Whitehead product of $\beta$ and $\gamma$ for $\beta \in \pi_{t}(X)$ and $\gamma \in \pi_{s}(X)$. Furthermore, we denote by $Z\{\nu\}$ the infinite cyclic group generated by $\nu$. For example, $\pi_{m}\left(S^{m}\right)=Z\left\{\iota_{m}\right\}$.

In our case, it is well-known that the homotopy set $[K, K]$ becomes a (noncommutative) ring if $n \geqq k+3$, and we are mainly concerned with studyig the ring structure of it because the group $\mathrm{Eq}(K)$ is the group consisting of all invertible elements of the ring $[K, K]$.

On the other hand, from the view-point of applications, we consider the homotopy type classification of highly-connected manifolds, in particular, the connected sums of sphere bundles over a sphere.

In fact, since such a manifold $M$ has a cell-decomposition with the form $K \bigcup_{\beta} e^{2 n+k+1}$ for $\beta \in \pi_{2 n+k}(K)$ up to homotopy, it is very important to investigate the group $\mathrm{Eq}(K)$ for this purpose.

Then the results of this paper are as follows:

Received January 19, 1983 
First, the additive group structure of $[K, K]$ is given by

THEOREM A.

$$
[K, K]=\bigoplus_{h, s=1}^{r} Z\left\{\bar{\sigma}_{h s}, \bar{\lambda}_{h s}\right\} \oplus G \cong 2 r^{2} Z \oplus G,
$$

where

and

$$
G=\bigoplus_{h=1}^{r} \pi_{h}^{*} \bar{G}_{h} \cong \bigoplus_{h=1}^{r} \bar{G}_{h}
$$

$$
\bar{G}_{h}=p_{h}^{*} l_{*} \pi_{n+k+1}\left(\bigvee^{r} S^{n}\right) / p_{h}^{*}\left(\bigoplus_{s \neq n} \jmath_{s^{*} l} s^{*} \alpha_{h} \pi_{n+k+1}\left(S^{n+k}\right)\right) .
$$

In particular, we have

COROLLARY B. If $n \geqq k+3$, then there exists an isomorphism

$$
[K, K] \cong \operatorname{Mat}(r, Z) \oplus \operatorname{Mat}(r, Z) \oplus \operatorname{Mat}\left(r, G_{h s}\right) \text {. }
$$

Secondly, we investigate the multiplicative structure of $[K, K]$ defined by the composition of maps, and we have

Theorem C. Let $\Theta$ and $\Theta^{\prime}$ be any elements of $[K, K]$ of the form (See Theorem A):

$$
\begin{aligned}
& \Theta=\sum_{h, s=1}^{r}\left(a_{h s} \bar{\sigma}_{h s}+b_{h s} \bar{\lambda}_{h s}\right)+g, \\
& \Theta^{\prime}=\sum_{h, s=1}^{r}\left(a_{h s}^{\prime} \bar{\sigma}_{h s}+b_{h s}^{\prime} \bar{\lambda}_{h s}\right)+g^{\prime}, \\
& g=\sum_{h=1}^{r} g_{h}, g^{\prime}=\sum_{h=1}^{r} g_{h}^{\prime \prime} \in G=\bigoplus_{h=1}^{r} \pi_{h}^{*} \bar{G}_{h}, \\
& g_{h}=\pi{ }_{h}^{*}\left(p_{h}^{*} i_{*}\left(\hat{g}_{h}\right)\right), g_{h}^{\prime \prime}=\pi_{h}^{*}\left(p_{h}^{*} \imath_{*}\left(\hat{g}_{h}^{\prime \prime}\right)\right) \in \pi_{h}^{*} \bar{G}_{h}, \\
& a_{h s}, b_{h s}, a_{h s}^{\prime}, b_{h s}^{\prime} \in Z \quad \text { for } 1 \leqq h, s \leqq r .
\end{aligned}
$$

Then the multiplication of $[K, K]$ is given by the formula:

$$
\Theta \circ \Theta^{\prime}=\sum_{h, s=1}^{r}\left(A_{h s} \bar{\sigma}_{h s}+B_{h s} \bar{\lambda}_{h s}\right)+\sum_{h=1}^{r} \pi_{h}^{*}\left(\Gamma_{h}\right)
$$

where

$$
\begin{aligned}
A_{h s}= & \sum_{q=1}^{r}\left(a_{h q}^{\prime} a_{q s} t_{s q} t_{q s} / t_{s h}\right), \\
B_{h s}= & \sum_{q=1}^{r}\left\{a_{h q}^{\prime} a_{q s}\left(t_{s h} t_{h q} t_{q s}-t_{h s} t_{s q} t_{q h}\right) /\left(m_{s} t_{s q}\right)\right. \\
& \left.+a_{h q}^{\prime} b_{q s} t_{h q}+\left(b_{h q}^{\prime} a_{q s} m_{q} t_{q s} / m_{s}\right)+b_{h q}^{\prime} b_{q s} m_{q}\right\},
\end{aligned}
$$


and

$$
\begin{aligned}
\Gamma_{h}= & \sum_{q=1}^{r}\left(a_{h q}^{\prime} t_{h q}+b_{h q^{\prime}}^{\prime} m_{q}\right) p_{h}^{*} l_{*}\left(\hat{g}_{q}\right) \\
& +\sum_{q, s=1}^{r}\left\{a_{h q}^{\prime} a_{q s} j_{s^{*}}\left(\gamma_{h s}^{q}\right)+b_{h q}^{\prime} a_{q s} j_{s^{*}}\left(\beta_{h s}^{q}\right)\right\} \\
& +p_{h}^{*}\left(\sum_{q, s=1}^{r} a_{q s} t_{s q} \jmath_{s^{*}} l_{s^{*}}\left(\pi_{h}^{\prime}\right)\right) \circ \hat{g}_{h}^{\prime \prime} .
\end{aligned}
$$

In the general situation, the above multiplication does not necessarily satisfy the left distributive law

$$
(\alpha+\beta) \circ \gamma=\alpha \circ \gamma+\beta \circ \gamma \quad(\alpha, \beta, \gamma \in[K, K]) .
$$

However, if $n \geqq k+3$, this one holds. So the homotopy set $[K, K]$ becomes a (non-commutative) ring.

Its ring structure is described by the following: put

$$
\Theta=\left(\theta_{h s}\right) \in \operatorname{Mat}\left(r,\left[K_{h}, K_{s}\right]\right)
$$

and

where

$$
\Theta^{\prime}=\left(\theta_{h s}^{\prime}\right) \in \operatorname{Mat}\left(r,\left[K_{h}, K_{s}\right]\right)
$$

$$
\begin{aligned}
& \theta_{h s}=a_{h s} \sigma_{h s}+b_{h s} \lambda_{h s}+g_{h s} \in\left[K_{h}, K_{s}\right], \\
& \theta_{h s}^{\prime}=a_{h s}^{\prime} \sigma_{h s}+b_{h s}^{\prime} \lambda_{h s}+g_{h s}^{\prime} \in\left[K_{h}, K_{s}\right], \\
& g_{h s}=p_{h}^{*} i_{s^{*}}\left(\hat{g}_{h s}\right) \in G_{h s}, \\
& g_{h s}^{\prime}=p_{h}^{*} i_{s^{*}}\left(\hat{g}_{h s}^{\prime}\right) \in G_{h s}, \\
& a_{h s}, a_{h s}^{\prime}, b_{h s}, b_{h s}^{\prime} \in Z \quad \text { for } 1 \leqq h, s \leqq r .
\end{aligned}
$$

Then the multiplicative structure of $[K, K]$ is given by the formula:

$$
\Theta \circ \Theta^{\prime}=\left(\theta_{h s}^{\prime \prime}\right) \in[K, K] \cong \operatorname{Mat}\left(r,\left[K_{h}, K_{s}\right]\right),
$$

where

$$
\theta_{h s}^{\prime \prime}=A_{h s} \sigma_{h s}+B_{h s} \lambda_{h s}+\Gamma_{h s} \in\left[K_{h}, K_{s}\right],
$$

and

$$
A_{h s} \text { and } B_{h s} \text { are defined by (4.22), }
$$




$$
\begin{aligned}
\Gamma_{h s}= & \sum_{q=1}^{r}\left\{\left(a_{h q}^{\prime} t_{h q}+b_{h q}^{\prime} m_{q}\right) p_{h s^{*}}^{*}\left(\hat{g}_{q s}\right)\right. \\
& \left.+a_{q s} t_{s q} p_{h{ }^{*}}^{*} i_{s^{*}}\left(\hat{g}_{h q}^{\prime}\right)+b_{h q}^{\prime} a_{q s} \beta_{h s}^{q}+a_{h q}^{\prime} a_{q s} \gamma_{h s}^{q}\right\} \\
& \text { for } 1 \leqq h, s \leqq r .
\end{aligned}
$$

Now we suppose that $\alpha_{h}=\alpha_{1}$ for $1 \leqq h \leqq r$. Then the above result can be simplified as follows:

THEOREM E. Let $n \geqq k+3$, and we suppose that

Then

$$
\alpha_{h}=\alpha_{1} \quad \text { for } 1 \leqq h \leqq r .
$$

where

$$
[K, K] \cong M\left(r ; m_{1}, G_{1}\right) \quad \text { as a } r i n g \text {, }
$$

the integer $m_{1}$ is the order of $\alpha_{1}$ and

$$
G_{1}=\pi_{n+k+1}\left(S^{n}\right) / \alpha_{1 *} \pi_{n+k+1}\left(S^{n+k}\right)=\pi_{n+k+1}\left(S^{n}\right) / E \alpha_{1}^{*} \pi_{n+1}\left(S^{n}\right) .
$$

We denote by $\operatorname{Inv}(M)$ the group of all multiplicative invertible elements of a ring $M$.

Then the above theorem implies

COROLLARY F. Under the same assumptions as above, we have an isomorphism

$$
\operatorname{Eq}(K) \cong \operatorname{Inv}\left(M\left(r ; m_{1}, G_{1}\right)\right) \text {. }
$$

For example, we can determine the group $\operatorname{Eq}\left(\bigvee^{r}\left(S^{n} \vee S^{n+m}\right)\right)$ for $n \geqq m+2$, and $\mathrm{Eq}\left(\stackrel{r}{V}^{m} C P^{2}\right)$ for $m \geqq 2$.

Finally, as an application we study the homotopy classification of connected sums of sphere bundles over a sphere which admit cross-sections.

Theorem G (H. Ishimoto, [3]). Let $n \geqq k+3$ and $k \geqq 1$. Let $X_{h}$ and $X_{h}^{\prime}$ be $n$-sphere bundles over $a(n+k+1)$-sphere which admit cross-sections, for $1 \leqq h \leqq r$.

Then the connected sums $\mathbb{W}_{h=1}^{r} X_{h}$ and $\#_{h=1}^{r} X_{h}^{\prime}$ are of the same homotopy type if and only if there exists an unimodular $(r \times r)$-matrix $A \in \mathrm{GL}(r, Z)$ such that

$$
\left(\begin{array}{c}
\lambda\left(X_{1}^{\prime}\right) \\
\vdots \\
\lambda\left(X_{r}^{\prime}\right)
\end{array}\right)=A \cdot\left(\begin{array}{c}
\lambda\left(X_{1}\right) \\
\vdots \\
\lambda\left(X_{r}\right)
\end{array}\right)
$$

where the abelian group $J \pi_{n+k}(S O(n)) / P \pi_{n+k+1}\left(S^{n}\right)$ is considered as a left $Z$ module. 
The above result has been already established by $\mathrm{H}$. Ishimoto ([3]) and he also considered the more general cases. However, his method is quite different from ours, and he used essentially the technique of the handlebody theory to prove it. On the contrary, we use simply the elementary homotopy theory, so our technique is applicable to the case of the connected sum of spherical fibrations over a sphere with a cross-section which do not always have the homotopy types of closed smooth manifolds, and we will treat it in the subsequent paper.

This paper is organized as follows: In $\S 2$ we construct several homotopy classes of maps needed to determine the structure of $\left[K_{h}, K_{s}\right]$ and $[K, K]$, and in $\S 3$ we determine the additive structure of them. Similarly, in $\S 4$ and $\S 5$, we determine the multiplicative structute of them. In $\S 6$ we classify the homotopy type of connected sums of sphere bundles over a sphere which admit crosssections.

In the final of this section, the author would like to take this opportunity to thank Professor S. Sasao for his sincere many valuable suggestions and encouragements.

\section{$\S 2$. The construction of elements of $\left[K_{h}, K_{s}\right]$.}

For each $1 \leqq h \leqq r$, let $K_{h}$ denote a $C W$ complex

$$
K_{h}=S^{n} \bigcup_{\alpha_{n}} e^{n+k+1}, \quad n \geqq 3, k \geqq 1,
$$

such that the attaching class $\alpha_{h} \in \pi_{n+k}\left(S^{n}\right)$ satisfying the following condition:

(2.2) There is an element $\alpha_{h}^{\prime \prime} \in \pi_{n+k-2}\left(S^{n-2}\right)$ such that $\alpha_{h}$ is a double suspension of $\alpha_{h}^{\prime \prime}, \alpha_{h}=E^{2} \alpha_{h}^{\prime \prime}$, and both $\alpha_{h}^{\prime}=E \alpha_{h}^{\prime \prime}$ and $\alpha_{h}$ have the same order $m_{h}$.

Then it is easy to see that the integer $m_{h}$ is finite and $\alpha_{h}=0$ for $n=3$.

Now let us define two complexes $K_{h}^{\prime}$ and $K_{h}^{\prime \prime}$ as follows :

$$
K_{h}^{\prime}=S^{n-1} \bigcup_{\alpha_{h}^{\prime}} e^{n+k} \text { and } K_{h}^{\prime \prime}=S^{n-2} \bigcup_{\alpha_{h}^{\prime}} e^{n+k-1} .
$$

Then for each $1 \leqq h \leqq r$, there is a cofibre sequence

$$
\begin{gathered}
S^{n+k-2} \stackrel{\alpha_{h}^{\prime \prime}}{\longrightarrow} S^{n-2} \stackrel{i_{h}^{\prime \prime}}{\longrightarrow} K_{h}^{\prime \prime} \stackrel{p_{h}^{\prime \prime}}{\longrightarrow} S^{n+k-1} \stackrel{\alpha_{h}^{\prime}}{\longrightarrow} S^{n-1} \\
\stackrel{i_{h}^{\prime}}{\longrightarrow} K_{h}^{\prime} \stackrel{p_{h}^{\prime}}{\longrightarrow} S^{n+k} \stackrel{\alpha_{h}}{\longrightarrow} S^{n} \stackrel{\imath_{h}}{\longrightarrow} K_{h} \stackrel{p_{h}}{\longrightarrow} S^{n+k+1} .
\end{gathered}
$$

Since $\alpha_{h}=E \alpha_{h}^{\prime}$ and $\alpha_{h}^{\prime}=E \alpha_{h}^{\prime \prime}$, we may canonically identify

$$
K_{h}=E K_{h}^{\prime} \quad \text { and } \quad K_{h}^{\prime}=E K_{h}^{\prime \prime} .
$$

Furthermore the following relations are clear. 


$$
\begin{aligned}
& \imath_{h}=E i_{h}^{\prime}, \quad i_{h}^{\prime}=E i_{h}^{\prime \prime}, \\
& p_{h}=-E p_{h}^{\prime}, \quad p_{h}^{\prime}=-E p_{h}^{\prime \prime}, \\
& \imath d_{h}=E \imath d_{h}^{\prime}, \quad \imath d_{h}^{\prime}=E \imath d_{h}^{\prime \prime},
\end{aligned}
$$

where $\imath d_{h}$, $i d_{h}^{\prime}$ and $\imath d_{h}^{\prime \prime}$ denote the homotopy classes of the identity maps $\imath d_{K_{h}}$, $\imath d_{K_{h}^{\prime}}$ and $i d_{K_{h}^{\prime}}$, respectively.

Here, for our purpose, we quote the well-known

LEMMA 2.7 (H. Toda, [12]).

(a) There is an element $\xi_{h}^{\prime} \in \pi_{n+k}\left(K_{h}^{\prime}\right)$ such that $p_{h *}^{\prime}\left(\xi_{h}^{\prime}\right)=m_{h \ell_{n+k}}$ and the element $\xi_{h}^{\prime}$ is determined up to $i_{h *}^{\prime} \pi_{n+k}\left(S^{n-1}\right)$. The element $\xi_{h}^{\prime}$ is called the coextension map of $m_{h} \iota_{n+k-1}$.

(b) There is an element $\beta_{h}^{\prime} \in\left[K_{h}^{\prime}, S^{n-1}\right]$ such that $\beta_{h}^{\prime} \circ i_{h}^{\prime}=m_{h}{ }_{n-1}$, and the element $\beta_{h}^{\prime}$ is determined up to $p_{n}^{\prime}{ }^{*} \pi_{n+k}\left(S^{n-1}\right)$. The element $\beta_{h}^{\prime}$ is called the extension map of $m_{h^{\imath} n-1}$.

For each $1 \leqq h \leqq r$, we define two elements $\xi_{h}$ and $\beta_{h}$ by

$$
\xi_{h}=-E \xi_{h}^{\prime} \in \pi_{n+k+1}\left(K_{h}\right),
$$

and

$$
\beta_{h}=E \beta_{h}^{\prime} \in\left[K_{h}, S^{n}\right] .
$$

Then it follows from Lemma 2.7 and (2.8) that we have

LEMMA 2.9. The above elements satisfy the following relations:

(a) $p_{h} \circ \xi_{h}=m_{h \ell_{n+k+1}}$.

(b) $\beta_{h} \circ i_{h}=m_{h} \iota_{h}$.

Similarly, we define two elements $\lambda_{h s}^{\prime}$ and $\mu_{h s}^{\prime}$ for $1 \leqq h, s \leqq r$ by

$$
\lambda_{h s}^{\prime}=\xi_{s}^{\prime} \circ p_{h}^{\prime}, \text { and } \mu_{h s}^{\prime}=i_{s}^{\prime} \circ \beta_{h}^{\prime} \quad \text { in }\left[K_{h}^{\prime}, K_{s}^{\prime}\right] \text {. }
$$

In particular, for $h=s$, we put

$$
\lambda_{h}^{\prime}=\lambda_{h h}^{\prime}, \text { and } \mu_{h}^{\prime}=\mu_{h h}^{\prime} .
$$

Furthermore, we define two elements $\lambda_{h s}$ and $\mu_{h s}$ for $1 \leqq h, s \leqq r$ by

$$
\lambda_{h s}=E \lambda_{h s}^{\prime} \text {, and } \mu_{h s}=E \mu_{h s}^{\prime} \quad \text { in }\left[K_{h}, K_{s}\right] \text {. }
$$

For $h=s$, we put

$$
\lambda_{h}=\lambda_{h h}, \text { and } \mu_{h}=\mu_{h h} .
$$

Then we have easily 
LEMMA 2.14.

$$
\begin{aligned}
& \text { (a) } \lambda_{h s}=\xi_{s} \circ p_{h} . \\
& \text { (b) } \mu_{h s}=i_{s} \circ \beta_{h} .
\end{aligned}
$$

Let us consider the relation between the attaching classes of $K_{h}^{\prime}, \alpha_{h}^{\prime} \in$ $\in \pi_{n+k-1}\left(S^{n-1}\right)$, for $1 \leqq h \leqq r$. For each $\beta \in \pi_{n+k-1}\left(S^{n-1}\right)$, we denote by $(\beta)$ the cyclic group generated by $\beta$. Then there is an element $\gamma \in \pi_{n+k-1}\left(S^{n-1}\right)$ satisfying $\left(\alpha_{h}^{\prime}\right) \cap\left(\alpha_{s}^{\prime}\right)=(\gamma)$. Thus, there is an uniquely determined pair of positive integers $\left(t_{h s}, t_{s h}\right)$ such that, if $\alpha_{h}^{\prime} \neq \alpha_{s}^{\prime}$, then

$$
\begin{aligned}
& \text { (i) } t_{h s} \alpha_{s}^{\prime}=t_{s h} \alpha_{h}^{\prime}=\gamma \text {, and } \\
& \text { (ii) } 1 \leqq t_{h s} \leqq m_{s}, \quad 1 \leqq t_{s h} \leqq m_{h} .
\end{aligned}
$$

In particular, if $\alpha_{h}^{\prime}=\alpha_{s}^{\prime}$, we put

$$
t_{h s}=t_{s h}=1 \text {. }
$$

LEMMA 2.17. There is an element $\sigma_{h s}^{\prime} \in\left[K_{h}^{\prime}, K_{s}^{\prime}\right]$ satisfying the conditions $\sigma_{h s}^{\prime} i_{h}^{\prime}=t_{s h} i_{s}^{\prime}$ and $p_{s}^{\prime} \circ \sigma_{h s}^{\prime}=t_{h s} p_{h}^{\prime}$. In particular, if $\alpha_{h}^{\prime}=\alpha_{s}^{\prime}$, then we can choose $\sigma_{h s}^{\prime}=i d_{h}^{\prime}$.

Proof. Since $\left[K_{h}^{\prime}, S^{n+k}\right] \cong \pi_{n+k}\left(S^{n+k}\right)$, the proof can be easily obtained.

Q.E.D.

Now we define the element $\sigma_{h s} \in\left[K_{h}, K_{s}\right]$ by

$$
\sigma_{h s}=E \sigma_{h s}^{\prime} .
$$

Then the following is obvious.

$$
\sigma_{h s^{\circ} \imath_{h}}=t_{s h} l_{s} \text {, and } p_{s^{\circ}} \sigma_{h s}=t_{h s} p_{h} .
$$

Since $K_{h}^{\prime}=E K_{h}^{\prime \prime}$, the homotopy set $\left[K_{h}^{\prime}, K_{s}^{\prime}\right]$ becomes a group with the track addition.

Here we note that the integer $m_{s} t_{s h}$ is divisible by $m_{h}$ and we obtain

Proposition 2.20. For each tripple of elements $\left(\sigma_{h s}^{\prime}, \xi_{h s}^{\prime}, \beta_{h s}^{\prime}\right)$, the following relation holds in $\left[K_{h}^{\prime}, K_{s}^{\prime}\right]$ :

$$
t_{h s} \lambda_{h s}^{\prime}+\left(m_{s} t_{s h} / m_{h}\right) \mu_{h s}^{\prime} \equiv m_{s} \sigma_{h s}^{\prime} \quad\left(\bmod G_{h s}^{\prime}\right),
$$

where $G_{h s}^{\prime}=p_{h}^{\prime} * i_{s *}^{\prime} \pi_{n+k}\left(S^{n-1}\right)$.

In particular, if $\alpha_{h}^{\prime}=\alpha_{s}^{\prime}$, then we can choose $\sigma_{h s}^{\prime}=i d_{h}^{\prime}$, so we have

Corollary 2.21 (S. Oka, [6]). For each coextension $\xi_{h}^{\prime}$ (resp. extension $\beta_{h}^{\prime}$ ). there exists an extension $\beta_{h}^{\prime}$ (resp. a coextension $\xi_{h}^{\prime}$ ) such that 
ON THE SELF-HOMOTOPY EQUIVALENCES

$$
\lambda_{h}^{\prime}+\mu_{h}^{\prime}=m_{h} \imath d_{h}^{\prime} \quad \text { in }\left[K_{h}^{\prime}, K_{h}^{\prime}\right] .
$$

Proof of Proposition 2.20. We put $x=t_{h s}, y=m_{s} t_{s h} / m_{h}$, and $z=m_{s}$. First, consider the exact sequence

Since

$$
\pi_{n+k}\left(K_{s}^{\prime}\right) \stackrel{p_{h}^{\prime} *}{\longrightarrow}\left[K_{h}^{\prime}, K_{s}^{\prime}\right] \stackrel{i_{h}^{\prime} *}{\longrightarrow} \pi_{n-1}\left(K_{s}^{\prime}\right) .
$$

$$
\begin{aligned}
i_{h}^{\prime} *\left(x \lambda_{h s}^{\prime}+y \mu_{h s}^{\prime}-z \sigma_{h s}^{\prime}\right) & =x\left(\xi_{s}^{\prime} \circ p_{h}^{\prime} \circ i_{h}^{\prime}\right)+y\left(i_{s}^{\prime} \circ \beta_{h}^{\prime} \circ i_{h}^{\prime}\right)-z\left(\sigma_{h s}^{\prime} \circ i_{h s}^{\prime}\right) \\
& =\left(y m_{h}\right) i_{s}^{\prime}-\left(z t_{s h}\right) i_{s}^{\prime} \\
& =0
\end{aligned}
$$

there is an element $\gamma \in \pi_{n+k}\left(K_{s}^{\prime}\right)$ satisfying

$$
x \lambda_{h s}^{\prime}+y \mu_{h s}^{\prime}-z \sigma_{h s}^{\prime}=p_{h}^{\prime} *(\gamma) .
$$

Secondly, consider the isomorphism

Since

$$
p_{h}^{\prime *}: \pi_{n+k}\left(S^{n+k}\right) \stackrel{\cong}{\longrightarrow}\left[K_{h}^{\prime}, S^{n+k}\right] .
$$

$$
\begin{aligned}
p_{h}^{\prime} *\left(p_{s}^{\prime} \circ \gamma\right) & =p_{s *}^{\prime}\left(p_{h}^{\prime *}(\gamma)\right) \\
& =p_{s *}^{\prime}\left(x \lambda_{h s}^{\prime}+y \mu_{h s}^{\prime}-z \sigma_{h s}^{\prime}\right) \\
& =x\left(p_{s}^{\prime} \circ \xi_{s}^{\prime} \circ p_{h}^{\prime}\right)+y\left(p_{s}^{\prime} \circ i_{s}^{\prime} \circ \beta_{h}^{\prime}\right)-s\left(p_{s}^{\prime} \circ \sigma_{h s}^{\prime} ;\right. \\
& =\left(x m_{s}\right) p_{h}^{\prime}-\left(z t_{h s}\right) p_{h}^{\prime} \\
& =0
\end{aligned}
$$

we have $p_{s}^{\prime}(\gamma)=0$. If we consider the exact sequence

then we obtain

$$
\pi_{n+k}\left(S^{n-1}\right) \stackrel{i_{s *}^{\prime}}{\longrightarrow} \pi_{n+k}\left(K_{s}^{\prime}\right) \stackrel{p_{s *}^{\prime}}{\longrightarrow} \pi_{n+k}\left(S^{n+k}\right)
$$

$$
x \lambda_{h s}^{\prime}+y \mu_{h s}^{\prime}-z \sigma_{h s}^{\prime}=p_{h}^{\prime *}(\gamma) \equiv 0 \quad\left(\bmod G_{h s}^{\prime}\right) .
$$

Thus the proof is completed.

Q.E.D.

Now, by applying the suspension functor homomorphism, Proposition 2.20 can be transformed into

Proposition 2.22. For each triple of elements $\left(\sigma_{h s}, \xi_{h}, \beta_{h}\right)$, the following relation holds in $\left[K_{h}, K_{s}\right]$ :

$$
t_{h s} \lambda_{h s}+\left(m_{s} t_{s h} m_{h}\right) \mu_{h s} \equiv m_{s} \sigma_{h s} \quad\left(\bmod G_{h s}\right),
$$

where the group $G_{h s}$ is defined in (2.26). 
Corollary 2.23 (S. Oka, [6]). For each extension $\beta_{h}$ (resp. coextension $\xi_{h}$ ), there is a coextension $\xi_{h}$ (resp. an extension $\beta_{h}$ ) such that

$$
\lambda_{h}+\mu_{h}=m_{h} \imath d_{h} \quad \text { in }\left[K_{h}, K_{h}\right] .
$$

Since $K_{h}=E^{2} K_{h}^{\prime \prime}$, the track addition defines an abelian group structure on the homotopy set $\left[K_{h}, K_{s}\right]$ for $1 \leqq h, s \leqq r$. Now we determine the additive structure of $\left[K_{h}, K_{s}\right]$.

First, we need the following two lemmas.

LEMMA 2.24.

$$
\pi_{n+k+1}\left(K_{s}\right)=Z\left\{\xi_{s}\right\} \oplus \imath_{s *} \pi_{n+k+1}\left(S^{n}\right) .
$$

Proof. Let $p r:\left(K_{s}, S^{n}\right) \rightarrow\left(S^{n+k+1}, s_{0}\right)$ be a natural projection map. Then it follows from the homotopy excision theorem that the induced homomorphism

$$
p r_{*}: \pi_{n+k+1}\left(K_{s}, S^{n}\right) \stackrel{\cong}{\longrightarrow} \pi_{n+k+1}\left(S^{n+k+1}\right)
$$

is an isomorphism. Hence we have the exact sequence

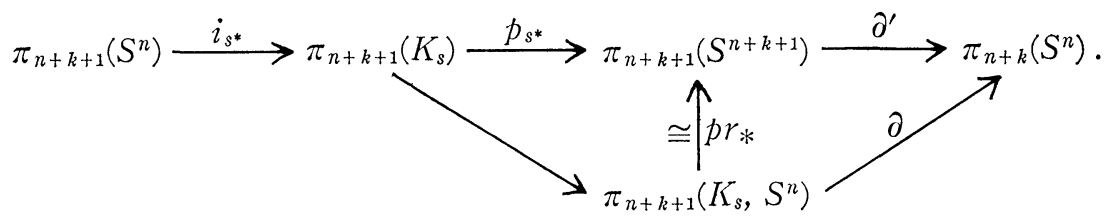

Let $\bar{\alpha}_{s}:\left(D^{n+k+1}, S^{n+k}\right) \rightarrow\left(K_{s}, S^{n}\right)$ be the characteristic map of the $(n+k+1)$-cell $e^{n+k+1}$ in $K_{s}$. Then it is clear that $\pi_{n+k+1}\left(K_{s}, S^{n}\right)=Z\left\{\bar{\alpha}_{s}\right\}$, and $p r_{*}\left(\bar{\alpha}_{s}\right)= \pm \iota_{n+k+1}$. Since $\partial\left(\bar{\alpha}_{s}\right)=\alpha_{s}$ and the order of $\alpha_{s}$ is $m_{s}$, we obtain $\operatorname{Im}\left(p_{s *}\right)=\left\{m_{s} \iota_{h+k+1}\right\} \cong Z$. Hence it follows (2.9) that we have

$$
\pi_{n+k+1}\left(K_{s}\right)=Z\left\{\xi_{s}\right\} \oplus \imath_{s *} \pi_{n+k+1}\left(S^{n}\right) .
$$

LEMma 2.25. Let E $\alpha_{h}^{*}$ denote the induced homomorphism

$$
E \alpha_{n}^{*}: \pi_{n+1}\left(K_{s}\right) \longrightarrow \pi_{n+k+1}\left(K_{s}\right) .
$$

Then $\operatorname{Im}\left(E \alpha_{h}^{*}\right)=i_{s *} \alpha_{h *} \pi_{n+k+1}\left(S^{n+k}\right)=\left\{i_{s} \circ \alpha_{h} \circ \eta_{n+k}\right\}$, where $\pi_{m+1}\left(S^{m}\right)=\left\{\eta_{m}\right\}=Z / 2 Z$ $=Z_{2}$ for $m \geqq 3$.

Proof. If $n=3$, then $\alpha_{h}=0$ and the statement is trivial. Otherwise, the proof easily follows from the Barratt-Hilton formula.

Q.E.D.

Then we have the following

Proposition 2.26. For each $1 \leqq h, s \leqq r$, 
ON THE SELF-HOMOTOPY EQUIVALENCES

where

$$
\left[K_{h}, K_{s}\right] \cong Z\left\{\sigma_{h s}\right\} \oplus Z\left\{\lambda_{h s}\right\} \oplus G_{h s} \cong Z \oplus Z \oplus G_{h s},
$$

$$
G_{h s}=p_{h}^{*} \imath_{s *} \pi_{n+k+1}\left(S^{n}\right) / p_{h}^{*} \imath_{s *} \alpha_{h *} \pi_{n+k+1}\left(S^{n+k}\right) .
$$

In particular, if $\alpha_{h}=\alpha_{s}$, we obtain

COROLlary 2.27 (S. Oka, [6]). For each $1 \leqq h \leqq r,\left[K_{h}, K_{h}\right] \cong Z\left\{\imath d_{h}\right\} \oplus Z\left\{\lambda_{h}\right\}$ $\oplus G_{h} \cong Z \oplus Z \oplus G_{h}$, where $G_{h}=p_{h}^{*} i_{h * \pi_{n+k+1}}\left(S^{n}\right)$.

Proof of Proposition 2.26. Consider the exact sequence

$$
\pi_{n+1}\left(K_{s}\right) \stackrel{E \alpha_{h}^{*}}{\longrightarrow} \pi_{n+k+1}\left(K_{s}\right) \stackrel{p_{h}^{*}}{\longrightarrow}\left[K_{h}, K_{s}\right] \stackrel{i_{h}^{*}}{\longrightarrow} \pi_{n}\left(K_{s}\right)=Z\left\{\imath_{s}\right\} .
$$

Applying (2.24) and (2.25) to the above sequence, we have the exact sequence

$$
0 \longrightarrow Z\left\{\xi_{s}\right\} \oplus \hat{G}_{h s}^{\prime} \stackrel{p_{h}^{*}}{\longrightarrow}\left[K_{h}, K_{s}\right] \stackrel{i_{h}^{*}}{\longrightarrow} Z\left\{\imath_{s}\right\} \stackrel{\alpha_{h}^{*}}{\longrightarrow} \pi_{n+k}\left(K_{s}\right),
$$

where $\hat{G}_{h s}^{\prime}=\imath_{s * \pi_{n+k+1}}\left(S^{n}\right) / \imath_{s *} \alpha_{h *} \pi_{n+k+1}\left(S^{n+k}\right)$.

On the other hand, since $t_{s h} \alpha_{h}=t_{h s} \alpha_{s}$ and $\operatorname{Ker}\left(\alpha_{h}^{*}\right)=\left\{t_{s h} \imath_{s}\right\} \cong Z$, the following sequence is exact.

$$
0 \longrightarrow Z\left\{\xi_{s}\right\} \oplus \hat{G}_{h s}^{\prime} \stackrel{p_{h}^{*}}{\longrightarrow}\left[K_{h}, K_{s}\right] \stackrel{i_{h}^{*}}{\longrightarrow}\left\{t_{s h} \imath_{s}\right\} \cong Z \longrightarrow 0 .
$$

Then the proof is completed by (2.19).

Q.E.D.

Here we remark the following result which is useful for the applications.

Proposition 2.28. Let $G_{h s}$ be the abelian group

Then

$$
p_{h}^{*} \imath_{s *} \pi_{n+k+1}\left(S^{n}\right) / p_{h}^{*} \imath_{s *} \alpha_{h * \pi_{n+k+1}}\left(S^{n+k}\right) .
$$

where

$$
G_{h s} \cong \pi_{n+k+1}\left(S^{n}\right) / I_{h s},
$$

and

$$
\begin{aligned}
& I_{h s}=\alpha_{h * \pi_{n+k+1}}\left(S^{n+k}\right)+\alpha_{s *} \pi_{n+k+1}\left(S^{n+k}\right), \\
& \alpha_{h * \pi_{n+k+1}}\left(S^{n+k}\right)=E \alpha_{h}^{*} \pi_{n+1}\left(S^{n}\right),
\end{aligned}
$$

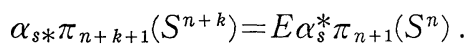

In particular, for the case $\alpha_{h}=\alpha_{s}$, we have

$$
\begin{aligned}
G_{h} & =G_{h h} \cong \pi_{n+k+1}\left(S^{n}\right) / \alpha_{h *} \pi_{n+k+1}\left(S^{n+k}\right) \\
& =\pi_{n+k+1}\left(S^{n}\right) / E \alpha_{h}^{*} \pi_{n+1}\left(S^{n}\right) .
\end{aligned}
$$

Proof. Consider the exact sequence 


$$
\pi_{n+1}\left(K_{s}\right) \stackrel{E \alpha_{h}^{*}}{\longrightarrow} \pi_{n+k+1}\left(K_{s}\right) \stackrel{p_{h}^{*}}{\longrightarrow}\left[K_{h}, K_{s}\right] .
$$

It follows from the Barratt-Hilton formula that the induced homomorphism

$$
p_{h}^{*}: i_{s *} \pi_{n+k+1}\left(S^{n}\right) / \imath_{s *} \alpha_{h *} \pi_{n+k+1}\left(S^{n+k}\right) \longrightarrow\left[K_{h}, K_{s}\right]
$$

is monic. Hence we have $G_{h s} \cong \imath_{s *} \pi_{n+k+1}\left(S^{n}\right) / \imath_{s *} \alpha_{h *} \pi_{n+k+1}\left(S^{n+k}\right)$. Next, consider the exact sequence

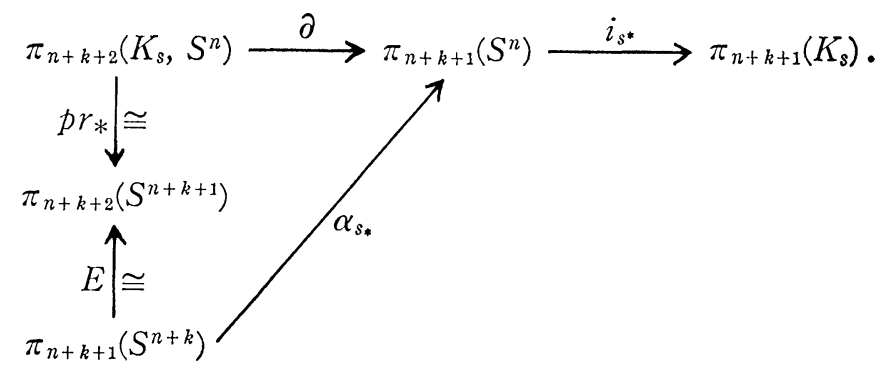

Then we have $i_{s *} \pi_{n+k+1}\left(S^{n}\right) \cong \pi_{n+k+1}\left(S^{n}\right) / \alpha_{s *} \pi_{n+k+1}\left(S^{n+k}\right)$, and the statement can be easily obtained.

Q.E.D.

\section{$\S 3$. The additive structure.}

Let $K, K^{\prime}$ and $K^{\prime \prime}$ denote the $C W$-complexes (See (2.3))

$$
\begin{aligned}
& K=\bigvee_{h=1}^{r} K_{h}, \\
& K^{\prime}=\bigvee_{h=1}^{r} K_{h}^{\prime},
\end{aligned}
$$

and

$$
K^{\prime \prime}=\bigvee_{h=1}^{r} K_{r}^{\prime \prime}
$$

Then it is easy to see that

$$
K=E K^{\prime}=E^{2} K^{\prime \prime} \text {, and } K^{\prime}=E K^{\prime \prime} .
$$

The aim of this section is to determine the additive structure of $[K, K]$, which is defined by the track addition.

Let $\alpha$ (resp. $\alpha^{\prime}$ ) denote the map

$$
\begin{gathered}
\alpha: V^{r} S^{n+k} \longrightarrow V^{r} S^{n} \\
\text { (resp. } \left.\alpha^{\prime}: V^{r} S^{n+k-1} \longrightarrow \bigvee^{r} S^{n-1}\right),
\end{gathered}
$$

where the $h$-th factor of $\alpha$ (resp. $\alpha^{\prime}$ ) is the map $\alpha_{h}$ (resp. $\alpha_{h}^{\prime}$ ). Since the com- 
plex $K$ (resp. $K^{\prime}$ ) is considered as the mapping cone of $\alpha$ (resp. $\alpha^{\prime}$ ), there is a cofibre sequence

$$
\begin{aligned}
& \stackrel{r}{V} S^{n+k-1} \stackrel{\alpha^{\prime}}{\longrightarrow} V^{r} S^{n-1} \stackrel{i^{\prime}}{\longrightarrow} K^{\prime} \stackrel{p^{\prime}}{\longrightarrow} V^{r} S^{n+k} \\
& \stackrel{\alpha}{\longrightarrow} \stackrel{r}{V}^{n} \stackrel{i}{\longrightarrow} K \stackrel{p}{\longrightarrow} \stackrel{r}{V}^{n+k+1} \stackrel{E \alpha}{\longrightarrow} V^{r} S^{n+1} .
\end{aligned}
$$

For each $1 \leqq h \leqq r$, let $j_{h}$ denote the natural inclusion map to the $h$-th factor

$$
j_{h}: K_{h} \longrightarrow K=\bigvee_{s=1}^{r} K_{s} .
$$

Then from $\pi_{n+1}(K)=2 * \pi_{n+1}\left(\vee^{\vee} S^{n}\right)$ and $\pi_{n+1}\left(\stackrel{r}{V}^{n}\right) \cong \stackrel{r}{\oplus} \pi_{n+1}\left(S^{n}\right)$, we obtain the following two lemmas.

LEMMA 3.6. $\pi_{n+k+1}(K)=2 * \pi_{n+k+1}\left(\bigvee^{r} S^{n}\right) \oplus\left(\bigoplus_{h=1}^{r} Z\left\{j_{h} \xi_{h}\right\}\right)$.

LEMMA 3.7. Let E $\alpha_{h}^{*}$ denote the induced homomorphism

Then,

$$
E \alpha_{n}^{*}: \pi_{n+1}(K) \longrightarrow \pi_{n+k+1}(K) .
$$

$$
\operatorname{Im}\left(E \alpha_{h}^{*}\right)=\bigoplus_{s \neq h} \jmath_{s * l} l_{s *} \alpha_{h * \pi_{n+k+1}\left(S^{n+k}\right)} .
$$

Thus, by replacing $K$ instead of $K_{s}$ in the proof of (2.26), we can obtain

PROPOSITION 3.8. For each $1 \leqq h \leqq r$,

$$
\left[K_{h}, K\right]==\bigoplus_{s=1}^{r} Z\left\{\jmath s^{\circ} \sigma_{h s}, \jmath_{s} \circ \lambda_{h s}\right\} \oplus G_{h} \cong 2 r Z \oplus G_{h},
$$

where

$$
G_{h}=p_{h}^{*} \imath_{*} \pi_{n+k+1}\left(\bigvee^{r} S^{n}\right) / p_{h}^{*}\left(\bigoplus_{s \neq n} \jmath_{s * l_{s *}} \alpha_{h * \pi_{n+k+1}}\left(S^{n+k}\right)\right) .
$$

Let $\pi_{h}: K=\bigvee_{s=1}^{r} K_{s} \rightarrow K_{h}$ be the natural projection map to the $h$-th factor. Then for each $1 \leqq h, s \leqq r$, we define two elements of $[K, K], \bar{\sigma}_{h s}$ and $\bar{\lambda}_{h s}$, by the following:

$$
\bar{\sigma}_{h s}=j_{s} \circ \sigma_{h s} \circ \pi_{h} \text { and } \bar{\lambda}_{h s}=\jmath_{s} \circ \lambda_{h s} \circ \pi_{h} .
$$

Then, from $[K, K]=\bigoplus_{h=1}^{r} \pi_{h}^{*}\left[K_{h}, K\right]$ and (3.8), we have

THEOREM A.

$$
[K, K]=\bigoplus_{h, s=1}^{r} Z\left\{\bar{\sigma}_{h s}, \bar{\lambda}_{h s}\right\} \oplus G \cong 2 r^{2} Z \oplus G,
$$


where

$$
G=\bigoplus_{h=1}^{r} \pi_{h}^{*} \bar{G}_{h} \cong \bigoplus_{h=1}^{r} \bar{G}_{h}
$$

and

$$
\bar{G}_{h}=p_{h}^{*} \imath_{*} \pi_{n+k+1}\left(\stackrel{r}{V}^{n}\right) / p_{h}^{*}\left(\bigoplus_{s \neq h} \jmath_{s *} i_{s *} \alpha_{h * \pi_{n+k+1}}\left(S^{n+k}\right)\right) .
$$

Now, for each $1 \leqq h, s \leqq r$, let $\Lambda_{h s}$ be an abelian group. Then we define the matrix group of degree $r$, Mat $\left(r, \Lambda_{h s}\right)$, by

$$
\operatorname{Mat}\left(r, \Lambda_{h s}\right)=\left\{A=\left(a_{h s}\right): a_{h s} \in \Lambda_{h s}\right\},
$$

where the $(h, s)$-entry of $A$ goes through $\Lambda_{h s}$. In particular, if $\Lambda_{h s}=\Lambda$ for any $(h, s)$, then $\operatorname{Mat}\left(r, \Lambda_{h s}\right)$ is the usual matrix group of degree $r$ with the coefficient $\Lambda$, $\operatorname{Mat}(r, \Lambda)$.

Let $\jmath$ denote the inclusion map

$$
\jmath: K=\bigvee_{h=1}^{r} K_{h} \longrightarrow \prod_{h=1}^{r} K_{h} .
$$

Proposition 3.12 (A.J. Sieradski, [10]). There is a split exact sequence

$$
0 \longrightarrow H \longrightarrow[K, K] \stackrel{\jmath *}{\longrightarrow} \operatorname{Mat}\left(r,\left[K_{h}, K_{s}\right]\right) \longrightarrow 0,
$$

where

$$
H \text { denotes the group } \bigoplus_{h=1}^{r}\left[C K_{h}, K_{h}: \prod_{s=1}^{r} K_{s}, K\right] \text {. }
$$

Proof. This follows from the exponential law and $K=E K^{\prime}$. See in detail $[10]$.

Q.E.D.

In general, it is difficult to compute the group $H$. However, if $n \geqq k+3, H$ is trivial by the dimensional reason and we have

Proposition 3.13. Let $n \geqq k+3$, then the induced homomorphism

$$
\jmath_{*}:[K, K] \stackrel{\cong}{\longrightarrow} \operatorname{Mat}\left(r,\left[K_{h}, K_{s}\right]\right)
$$

is an isomorphism.

Let $n \geqq k+3$. Then it follows from (3.13) that, for $\Theta \in[K, K]$, there is an element $A=\left(\theta_{h s}\right) \in \operatorname{Mat}\left(r,\left[K_{h}, K_{s}\right]\right)$ satisfying

$$
\jmath *(\Theta)=\left(\theta_{h s}\right)
$$

and this expression is uniquely determined. Since $\theta_{h s}$ is an element of $\left[K_{h}, K_{s}\right]$, by Proposition 2.26, there exists uniquely determined triple $\left(a_{h s}, b_{h s}, g_{h s}\right)$ such that, 


$$
\theta_{h s}=a_{h s} \sigma_{h s}+b_{h s} \lambda_{h s}+g_{h s},
$$

where

$$
a_{h s}, b_{h s} \in Z \text { and } g_{h s} \in G_{h s} .
$$

We may consider $\left(a_{h s}\right),\left(b_{h s}\right)$ and $\left(g_{h s}\right)$ as an element of $\operatorname{Mat}(r, Z)$, Mat $(r, Z)$ and $\operatorname{Mat}\left(r, G_{h s}\right)$, respectively, and Proposition 3.13 can be restated as

COROLlary B. If $n \geqq k+3$, then we have a group ısomorphism

$$
\jmath_{*}:[K, K] \longrightarrow \operatorname{Mat}(r, Z) \oplus \operatorname{Mat}(r, Z) \oplus \operatorname{Mat}\left(r, G_{h s}\right) .
$$

\section{$\S 4$. The multiplicative structure.}

The purpose of this section is to investigate the multiplicative structure of $[K, K]$ which is defined by the composition of maps. In particular, for the case $n \geqq k+3,[K, K]\left(\cong \operatorname{Mat}\left(r,\left[K_{h}, K_{s}\right]\right)\right)$ becomes a (non-commutative) ring and we will study its ring structure.

First, for each $1 \leqq h, s, m \leqq r$, we consider the multiplication

$$
\left[K_{h}, K_{s}\right] \times\left[K_{m}, K_{h}\right] \longrightarrow\left[K_{m}, K_{s}\right]
$$

which is also defined by the composition of maps. It is well-known that the left distributive law

$$
\beta \circ(\gamma+\delta)=\beta \circ \gamma+\beta \circ \delta
$$

holds in (4.1). But, in general, the right one does not hold. However, as is well known, we have

$$
(\gamma+\delta) \cdot E \beta=\gamma \circ E \beta+\delta \circ E \delta \quad \text { for } \beta \in[X, Y] \text { and } \gamma, \delta \in[E Y, Z] .
$$

In particular,

$$
\begin{aligned}
& a \gamma=\gamma \circ\left(a\left(\imath d_{X}\right)\right), \quad a E \gamma^{\prime}=\left(a\left(\imath d_{E Y}\right)\right) \circ E \gamma^{\prime} \\
& \quad \text { for } \gamma, \gamma^{\prime} \in[X, Y] \text { and any integer } a .
\end{aligned}
$$

Now, for each integer $a$, we define a homomorphism

$$
\phi_{a}^{s}:\left[K_{h}, K_{s}\right] \longrightarrow\left[K_{h}, K_{s}\right]
$$

by

$$
\phi_{a}^{s}(\theta)=\left(a\left(i d_{s}\right)\right) \circ \theta \quad \text { for } \theta \in\left[K_{h}, K_{s}\right] .
$$

Similarly, we define a homomorphism

$$
\phi_{a}: \pi_{n+k+1}\left(S^{n}\right) \longrightarrow \pi_{n+k+1}\left(S^{n}\right)
$$

by 


$$
\phi_{a}(g)=\left(a \iota_{n}\right) \circ g \quad \text { for } g \in \pi_{n+k+1}\left(S^{n}\right) .
$$

Then, from the formula (4.2) we have

LEMMA 4.6.

(a) $\phi_{a}^{s} \circ p_{h}^{*} \circ i_{s *}=p_{h}^{*} i_{s *^{\circ}} \phi_{s}$.

(b) $\phi_{a}^{s}(\theta)=a \theta \quad$ for $\theta=E \theta^{\prime} \in\left[K_{h}, K_{s}\right]$.

(c) $\phi_{a}(g)=a g \quad$ for $g=E g^{\prime} \in \pi_{n+k+1}\left(S^{n}\right)$.

Since $\sigma_{h s}=E \sigma_{h s}^{\prime}$ and $\lambda_{h s}=E \lambda_{h s}^{\prime}$, we have

$$
\phi_{a}^{s}\left(\sigma_{h s}\right)=a \sigma_{h s} \quad \text { and } \quad \phi_{a}^{s}\left(\lambda_{h s}\right)=a \lambda_{h s} .
$$

In particular, the above lemma shows that the subgroup $G_{h s}$ is closed with respect to $\phi_{a}^{s}$, and that $\phi_{a}^{s} \mid G_{h s}$ is determined by $\phi_{a}$.

PROPOSITION 4.8. Let

$$
\begin{aligned}
g=p_{h}^{*} i_{s *}\left(g_{1}\right) & \in G_{h s} \quad \text { and } \quad g^{\prime}=p_{m}^{*} i_{h *}\left(g_{1}^{\prime}\right) \in G_{m h} \\
\text { for } & g_{1} \in \pi_{n+k+1}\left(S^{n}\right) / \alpha_{h *} \pi_{n+k+1}\left(S^{n+k}\right) \text { and } \\
& g_{1}^{\prime} \in \pi_{n+k+1}\left(S^{n}\right) / \alpha_{m *} \pi_{n+k+1}\left(S^{n+k}\right) ;
\end{aligned}
$$

then the following relations hold:

(1) $\lambda_{h s} \circ \lambda_{m h}=m_{h} \lambda_{m s}$.

(2) $\lambda_{h s^{\circ}} \sigma_{m h}=t_{m h} \lambda_{m s}$.

(3) $\lambda_{h s^{\circ}} g^{\prime}=0$.

(4) $g \circ \lambda_{m h}=m_{h}\left(p_{m}^{*} i_{s *}\left(g_{1}\right)\right)$.

(5) $\sigma_{h s^{\circ}} g^{\prime}=\psi_{t_{s h}}\left(p_{m}^{*} i_{s *}\left(g_{1}^{\prime}\right)\right)$.

(6) $g \circ \sigma_{m h}=t_{m h}\left(p_{m}^{*} i_{s *}\left(g_{1}\right)\right)$.

(7) $g \circ g^{\prime}=0$.

Proof.

(1) $\lambda_{h s} \circ \lambda_{m h}=\left(\xi_{s} \circ p_{h}\right) \circ\left(\xi_{h} \circ p_{m}\right)$

$$
\begin{aligned}
& =\xi_{s} \circ\left(p_{h} \circ \xi_{h}\right) \circ p_{m} \\
& =\xi_{s} \circ\left(m_{h} \ell_{n+k+1}\right) \circ p_{m} \quad(\text { by }(2.9)) \\
& =m_{h}\left(\xi_{s} \circ p_{m}\right) \\
& =m_{h} \lambda_{m s} .
\end{aligned}
$$


(2) $\lambda_{h s^{\circ}} \sigma_{m h}=\left(\xi_{s} \circ p_{h}\right) \circ \sigma_{m h}$

$$
\begin{aligned}
& =\xi_{s} \circ\left(p_{h} \circ \sigma_{m h}\right) \\
& =\xi_{s} \circ\left(t_{m h} p_{m}\right) \\
& =t_{m n}\left(\xi_{s} \circ p_{m}\right) \\
& =t_{m h} \lambda_{m s} .
\end{aligned}
$$

(3) Since $p_{h} \circ i_{h}=0, \lambda_{h s^{\circ}} g^{\prime}=\left(\xi_{s} \circ p_{h}\right) \circ\left(i_{h} \circ g_{1}^{\prime} \circ p_{m}\right)=0$.

(4) $g \circ \lambda_{m h}=\left(i_{s} \circ g_{1} \circ p_{h}\right) \circ\left(\xi_{h} \circ p_{m}\right)$

$$
\begin{aligned}
& =i_{s} \circ g_{1} \circ\left(p_{h} \circ \xi_{h}\right) \circ p_{m} \\
& =i_{s} \circ g_{1} \circ\left(m_{h} \ell_{n+k+1}\right) \circ p_{m} \quad(\text { by }(2.9)) \\
& =m_{h}\left(p_{m}^{*} i_{s *}\left(g_{1}\right)\right) .
\end{aligned}
$$

The rest of the proof can be obtained by the similar argument.

Q.E.D.

PROPOSITION 4.9.

(a) $\sigma_{h s} \circ \lambda_{m h} \equiv\left(m_{h} t_{h s} / m_{s}\right) \lambda_{m s} \bmod G_{m s}$.

(b) $\sigma_{h s^{\circ}} \sigma_{m h} \equiv\left\{\left(t_{s m} t_{m h} t_{h s}-t_{m s} t_{s h} t_{h m}\right) / m_{s} t_{s m}\right\} \lambda_{m s}+\left(t_{s h} t_{h m} / t_{s m}\right) \sigma_{m s} \quad \bmod G_{m s}$.

Proof. It follows from Proposition 2.26 that we may assume

$$
\begin{aligned}
\sigma_{h s} \lambda_{m h}=a \lambda_{m s}+b \sigma_{m s}+g & \text { for } a, b \in Z \\
& \text { and } g=p_{m}^{*} i_{s *}\left(g^{\prime}\right) \in G_{m s} .
\end{aligned}
$$

Since

we have

$$
i_{m}^{*}\left(\sigma_{h s} \circ \lambda_{m h}\right)=\sigma_{h s} \circ \lambda_{m h} \circ i_{m}=\sigma_{h s} \circ \xi_{h} \circ p_{m} \circ i_{m}=0
$$

$$
\begin{aligned}
0 & =i_{m}^{*}\left(a \lambda_{m s}+b \sigma_{m s}+g\right) & & \\
& =\left(a \lambda_{m s}+b \sigma_{m s}+g\right) \circ i_{m} & & \\
& =a\left(\lambda_{m s} \circ i_{m}\right)+b\left(\sigma_{m s^{\circ} i_{m}}\right)+g \circ i_{m} & & \left(\text { by } i_{m}=E i_{m}^{\prime}\right) \\
& =a\left(\xi_{s} \circ p_{m} \circ i_{m}\right)+b\left(t_{s m} i_{s}\right)+\left(i_{s} \circ g^{\prime} \circ p_{m} \circ i_{m}\right) & & (\text { by }(2.19)) \\
& =\left(b t_{s m}\right) i_{s} . & & \left(\text { by } p_{m} \circ i_{m}=0\right)
\end{aligned}
$$

Hence $b=0$. Similarly, from (2.9) and (2.19) we have

$$
p_{s *}\left(\sigma_{h s} \circ \lambda_{m h}\right)=\left(p_{s} \circ \sigma_{h s}\right) \circ \lambda_{m h}=\left(t_{h s} p_{h}\right) \circ\left(\xi_{h} \circ p_{m}\right)=\left(m_{h} t_{h s}\right) p_{m} .
$$

On the other hand, by using $p_{s} \circ i_{s}=0$, 


$$
\begin{aligned}
p_{s *}\left(\sigma_{h s} \lambda_{m h}\right) & =p_{s *}\left(a \lambda_{m s}+g\right) \\
& =p_{s} \circ\left(a \xi_{s} \circ p_{m}\right) \\
& =\left(a m_{s}\right) p_{m} . \quad(\text { by }(2.9))
\end{aligned}
$$

Hence

and so

$$
\left(a m_{s}\right) p_{m}=\left(m_{h} t_{h s}\right) p_{m} \quad \text { in }\left[K_{m}, S^{n+k+1}\right]=Z\left\{p_{m}\right\},
$$

Therefore,

$$
a=m_{h} t_{h s} / m_{s} .
$$

$$
\sigma_{h s} \cdot \lambda_{m h}=\left(m_{h} t_{h s} / m_{s}\right) \lambda_{m s} \quad \bmod G_{h s} .
$$

Thus the statement (a) is established and (b) also can be shown by the similar argument.

Q.E.D.

The above proposition implies that, for each $1 \leqq m, h, s \leqq r$, there exists elements $\beta_{m s}^{h}$ and $\gamma_{m s}^{h} \in G_{m s}$ satisfying two conditions

$$
\sigma_{h s} \circ \lambda_{m h}=\left(m_{h} t_{h s} / m_{s}\right) \lambda_{m s}+\beta_{m s}^{h}
$$

and

$$
\begin{aligned}
\sigma_{h s^{\circ}} \sigma_{m h}= & \left\{\left(t_{s m} t_{m h} t_{h s}-t_{m s} t_{s h} t_{h m}\right) / m_{s} t_{s m}\right\} \lambda_{m s} \\
& +\left(t_{s h} t_{h m} / t_{s m}\right) \sigma_{m s}+\gamma_{m s}^{h} .
\end{aligned}
$$

In particular, we note that

$$
\beta_{m s}^{h}=0 \quad \text { if } \alpha_{h}=\alpha_{s}
$$

and

$$
\gamma_{m s}^{h}=0 \quad \text { if } \alpha_{h}=\alpha_{s} \text { or } \alpha_{m}=\alpha_{h} .
$$

THEOREM 4.14. The multiplication (4.1) is given by the formula:

$$
\begin{aligned}
\Theta \circ \Theta^{\prime}= & \left(a a^{\prime} t_{s h} t_{h m} / t_{s m}\right) \sigma_{m s} \\
& +\left\{a a^{\prime}\left(t_{s m} t_{m h} t_{h s}-t_{m s} t_{s h} t_{h m}\right) /\left(m_{s} t_{s m}\right)+\left(a b^{\prime} m_{h} t_{h s} / m_{s}\right)\right. \\
& \left.+a^{\prime} b t_{m h}+b b^{\prime} m_{h}\right\} \lambda_{m s} \\
& +\left(a^{\prime} t_{m h}+b^{\prime} m_{h}\right) p_{m}^{*} l_{s *}\left(g_{1}\right)+\phi_{a t_{s h}}^{s}\left(p_{m}^{*} l_{s *}\left(g_{1}^{\prime}\right)\right) \\
& +a a^{\prime} \gamma_{m s}^{h}+a b^{\prime} \beta_{m s}^{h}, \\
\text { for } \quad & \Theta=a \sigma_{h s}+b \lambda_{h s}+g \in\left[K_{h}, K_{s}\right] \quad \text { and } \\
& \Theta^{\prime}=a^{\prime} \sigma_{m h}+b^{\prime} \lambda_{m h}+g^{\prime} \in\left[K_{m}, K_{h}\right],
\end{aligned}
$$


where $a, a^{\prime}, b, b^{\prime} \in Z$,

and

$$
g=p_{h \imath_{s} *}^{*}\left(g_{1}\right) \in G_{h s},
$$

$$
g^{\prime} p_{m}^{*} \imath_{h *}\left(g_{1}^{\prime}\right) \in G_{m h} .
$$

In particular, for the case $h=s=m$, we also have

COROLlaRY 4.15 (S. Oka, [6]). The multiplication of $\left[K_{h}, K_{h}\right]$ is given by the formula:

$$
\begin{gathered}
\Theta \circ \Theta^{\prime}=a a^{\prime} \imath d_{h}+\left(a b^{\prime}+a^{\prime} b+m_{h} b b^{\prime}\right) \lambda_{h}+\left(a^{\prime}+b^{\prime} m_{h}\right) g+\phi_{a}^{h}\left(g^{\prime}\right) \\
\text { for } \Theta=a i d_{h}+b \lambda_{h}+g \in\left[K_{h}, K_{h}\right]
\end{gathered}
$$

and

$$
\Theta^{\prime}=a^{\prime} \imath d_{h}+b^{\prime} \lambda_{h}+g^{\prime} \in\left[K_{h}, K_{h}\right],
$$

where $a, a^{\prime}, b, b^{\prime} \in Z$ and $g, g^{\prime} \in G_{h}$.

Proof of (4.14). It follows from the right distributive law that

$$
\begin{aligned}
\Theta \circ \Theta^{\prime} & =\Theta \circ\left(a^{\prime} \sigma_{m h}+b^{\prime} \lambda_{m h}+g^{\prime}\right) \\
& =a^{\prime} \Theta \circ \sigma_{m h}+b^{\prime} \Theta \circ \lambda_{m h}+\Theta \circ g^{\prime} .
\end{aligned}
$$

Since $\sigma_{m h}=E \sigma_{m h}^{\prime}$, we have

$$
\begin{aligned}
a^{\prime} \Theta \circ \sigma_{m h}= & a a^{\prime} \sigma_{h s^{\circ}} \sigma_{m h}+a^{\prime} b \lambda_{h s}{ }^{\circ} \sigma_{m h}+a^{\prime}\left(g \circ \sigma_{m h}\right) \\
= & \left(a a^{\prime} t_{s h} t_{h m} / t_{s m}\right) \sigma_{m s}+a a^{\prime} \gamma_{m s}^{h} \\
& +\left\{a a^{\prime}\left(t_{s m} t_{m h} t_{h s}-t_{m s} t_{s h} t_{h m}\right) / m_{s} t_{s m}\right\} \lambda_{m s} \\
& +\left(a^{\prime} b t_{m h}\right) \lambda_{m s}+\left(a^{\prime} t_{m h}\right) p_{m}^{*} l_{s *}\left(g_{1}\right) . \\
= & \left(a a^{\prime} t_{s h} t_{h m} / t_{s m}\right) \sigma_{m s}+\left\{a a^{\prime}\left(t_{s m} t_{m h} t_{h s}-t_{m s} t_{s h} t_{h m}\right) /\right. \\
& \left.\left(m_{s} t_{s m}\right)+a^{\prime} b t_{m h}\right\} \lambda_{m s}+\left(a^{\prime} t_{m h}\right) p_{m}^{*} i_{s *}\left(g_{1}\right)+a a^{\prime} \gamma_{m s}^{h} .
\end{aligned}
$$

Similarly, by using $\lambda_{m h}=E \lambda_{m h}^{\prime}$, we have

$$
b^{\prime} \Theta \circ \lambda_{m h}=\left\{\left(a b^{\prime} m_{h} t_{h s} / m_{s}\right)+b b^{\prime} m_{h}\right\} \lambda_{m s}+\left(m_{h} b^{\prime}\right) p_{m}^{*} \imath_{s *}\left(g_{1}\right)+a b^{\prime} \beta_{m s}^{h} .
$$

Moreover, by using $i_{h}=E i_{h}^{\prime}$ we have

$$
\begin{aligned}
\Theta \circ g^{\prime} & =\left(a \sigma_{h s}+b \lambda_{h s}+g\right) \circ\left(l_{h} \circ g_{1}^{\prime} \circ p_{m}\right) \\
& =\left(a \sigma_{h s} \circ i_{h}+b \lambda_{h s^{\circ}} i_{h}+g \circ \imath_{h}\right) \circ\left(g_{1}^{\prime} \circ p_{m}\right) \\
& =\left(a t_{s h} i_{s}\right) \circ\left(g_{1}^{\prime} \circ p_{m}\right) \\
& =\phi_{a t_{s h}}^{s}\left(p_{m}^{*} i_{s *}\left(g_{1}^{\prime}\right)\right) .
\end{aligned}
$$


Thus the proof is completed.

Q.E.D.

Let $\pi_{h}^{\prime}$ denote the natural projection map to the $h$-th factor,

$$
\pi_{h}^{\prime}: \stackrel{r}{\vee} S^{n} \longrightarrow S^{n}
$$

Furthermore we put

$$
\hat{\beta}_{m s}^{h}=\pi_{m}^{\prime *} \jmath_{s *} \beta_{m s}^{h} \text { and } \hat{\gamma}_{m s}^{h}=\pi_{m}^{\prime} *_{s} * \gamma_{m s}^{h} .
$$

Now, we proceed to investigate the multiplication

$$
[K, K] \times[K, K] \longrightarrow[K, K]
$$

which is defined by the composition of maps.

Proposition 4.19. Let $g=\sum_{h=1}^{r} g_{h}$ and $g^{\prime}=\sum_{h=1}^{r} g_{h}^{\prime \prime}$ be any elements in $G=\bigoplus_{h=1}^{r} \pi_{h}^{*} \bar{G}_{h}$, where $g_{h}=\pi_{h}^{*}\left(p_{h}^{*} \imath_{*}\left(\hat{g}_{h}\right)\right)$ and $g_{h}^{\prime \prime}=\pi_{h}^{*}\left(p_{h}^{*} i_{*}\left(\hat{g}_{h}^{\prime \prime}\right)\right) \in \pi_{h}^{*} \bar{G}_{h}$ for $\hat{g}_{h}, \hat{g}_{h}^{\prime \prime} \in \pi_{n+k+1}\left(\bigvee^{r} S^{n}\right)$, $1 \leqq h \leqq r$.

Then the following relations hold:
(1) $\quad \bar{\lambda}_{h s} \circ \bar{\lambda}_{m q}=\left\{\begin{array}{l}m_{h} \bar{\lambda}_{m s} \\ 0\end{array}\right.$
if $h=q$
(2) $\bar{\lambda}_{h s^{\circ}} \bar{\sigma}_{m q}=\left\{\begin{array}{l}t_{m h} \bar{\lambda}_{m s} \\ 0\end{array}\right.$
otherwise.
(3) $\quad \bar{\sigma}_{h s} \circ \bar{\lambda}_{m q}=\left\{\begin{array}{l}\left(m_{h} t_{h s} / m_{s}\right) \bar{\lambda}_{m s}+\hat{\beta}_{m s}^{h} \\ 0\end{array}\right.$
if $h=q$
otherwise.
(4) $\quad \bar{\sigma}_{h s^{\circ}} \bar{\sigma}_{m q}=\left\{\begin{array}{cc}\left\{\left(t_{s m} t_{m h} t_{h s}-t_{m s} t_{s h} t_{h m}\right) / m_{s} t_{s m}\right\} \bar{\lambda}_{m s}+ \\ \left(t_{s h} t_{h m} / t_{s m}\right) \bar{\sigma}_{m s}+\hat{\gamma}_{m s}^{h} & \text { if } h=q \\ 0 & \text { otherwise. }\end{array}\right.$

(5) $\bar{\lambda}_{h s^{\circ}} g^{\prime}=0$.

(6) $g \circ \bar{\lambda}_{m q}=m_{q} \pi_{m}^{*}\left(p_{m}^{*} i_{*}\left(\hat{g}_{q}\right)\right)$.

(7) $\quad \bar{\sigma}_{h s^{\circ}} g^{\prime}=t_{s h}\left(\sum_{m=1}^{r} \pi_{m}^{*}\left(j_{s * l_{s} *} \pi_{h *}^{\prime} p_{m}^{*}\left(\hat{g}_{m}^{\prime \prime}\right)\right)\right)$.

(8) $g \circ \bar{\sigma}_{m q}=t_{m q} \pi_{m}^{*}\left(p_{m}^{*} \imath_{*}\left(\hat{g}_{q}\right)\right)$.

(9) $g \circ g^{\prime}=0$.

Proof. Since $\pi_{h} \circ j_{q}=\delta_{h q} i d_{h}$, the proof follows from (4.18), (4.10) and (4.11), 
where $\delta_{h q}$ denotes the Kronecker delta.

Q.E. D.

Now, the multiplication of $[K, K]$ is stated as follows :

TheOREM C. Let $\Theta$ and $\Theta^{\prime}$ be any elements of $[K, K]$ of the forms

$$
\begin{aligned}
& \Theta=\sum_{h, s=1}^{r}\left(a_{h s} \bar{\sigma}_{h s}+b_{h s} \bar{\lambda}_{h s}\right)+g, \\
& \Theta^{\prime}=\sum_{h, s=1}^{r}\left(a_{h s}^{\prime} \bar{\sigma}_{h s}+b_{h s}^{\prime} \bar{\lambda}_{h s}\right)+g^{\prime}, \\
& g=\sum_{h=1}^{r} g_{h}, \quad g^{\prime}=\sum_{h=1}^{r} g_{h}^{\prime \prime} \in G=\bigoplus_{h=1}^{r} \pi_{h}^{*} \bar{G}_{h}, \\
& g_{h}=\pi_{h}^{*}\left(p_{h}^{*} l_{*}\left(\hat{g}_{h}\right)\right), \quad g_{h}^{\prime \prime}=\pi_{h}^{*}\left(p_{h}^{*} l_{*}\left(\hat{g}_{h}^{\prime \prime}\right)\right) \in \pi_{h}^{*} \bar{G}_{h}, \\
& a_{h s}, b_{h s}, a_{h s}^{\prime}, b_{h s}^{\prime} \in Z \quad \text { for } 1 \leqq h, s \leqq r .
\end{aligned}
$$

Then the multiplication of $[K, K]$ is given by the formula:

$$
\Theta \circ \Theta^{\prime}=\sum_{h, s=1}^{r}\left(A_{h s} \bar{\sigma}_{h s}+B_{h s} \bar{\lambda}_{h s}\right)+\sum_{h=1}^{r} \pi_{h}^{*}\left(\Gamma_{h}\right),
$$

where

and

$$
\begin{aligned}
A_{h s}= & \sum_{q=1}^{r}\left(a_{h q}^{\prime} a_{q s} t_{s q} t_{q h} / t_{s h}\right), \\
B_{h s}= & \sum_{q=1}^{r}\left\{a_{h q}^{\prime} a_{q s}\left(t_{s h} t_{h q} t_{q s}-t_{h s} t_{s q} t_{q h}\right) /\left(m_{s} t_{s q}\right)\right. \\
& \left.+a_{h q}^{\prime} b_{q s} t_{h q}+\left(b_{h q}^{\prime} a_{q s} m_{q} t_{q s} / m_{s}\right)+b_{h q}^{\prime} b_{q s} m_{q}\right\},
\end{aligned}
$$

$$
\begin{aligned}
\Gamma_{h}= & \sum_{q=1}^{r}\left(a_{h q}^{\prime} t_{h q}+b_{h q}^{\prime} m_{q}\right) p_{h}^{*} \imath_{*}\left(\hat{g}_{q}\right) \\
& +\sum_{q, s=1}^{r}\left\{a_{h q}^{\prime} a_{q s} \jmath_{s *}\left(\gamma_{h s}^{q}\right)+b_{h q}^{\prime} a_{q s} \jmath_{s *}\left(\beta_{h s}^{q}\right)\right\} \\
& +p_{h}^{*}\left(\sum_{q, s=1}^{r} a_{q s} t_{s q} \jmath_{s *} l_{s *}\left(\pi_{h}^{\prime}\right)\right) \circ \hat{g}_{h}^{\prime \prime} .
\end{aligned}
$$

Proof. It follows from the right distributive law that

$$
\Theta \circ \Theta^{\prime}=\sum_{m, q=1}^{r} a_{m q}^{\prime} \Theta \circ \bar{\sigma}_{m q}+\sum_{m, q=1}^{r} \Theta \circ \bar{\lambda}_{m q}+\Theta \circ g^{\prime} .
$$

Since $\sigma_{m q}=E \sigma_{m q}^{\prime}$, by using (4.2)

$$
\Theta \circ \sigma_{m q}=\sum_{h, s=1}^{r} a_{h s} \bar{\sigma}_{h s^{\circ}} \bar{\sigma}_{m q}+\sum_{h, s=1}^{r} b_{h s} \bar{\lambda}_{h s^{\circ}} \bar{\sigma}_{m q}+g \circ \bar{\sigma}_{m q}
$$




$$
\begin{aligned}
= & \sum_{s=1}^{r} a_{q s}\left(t_{s q} t_{q m} / t_{s m}\right) \bar{\sigma}_{m s}+\sum_{s=1}^{r} a_{q s} \pi_{m}^{*} j_{s *}\left(\gamma_{m s}^{q}\right) \\
& +\sum_{s=1}^{r} a_{q s}\left\{\left(t_{s m} t_{m q} t_{q s}-t_{m s} t_{s q} t_{q m}\right) / m_{s} t_{s m}\right\} \bar{\lambda}_{m s} \\
& +\sum_{s=1}^{r} b_{q s} t_{m q} \bar{\lambda}_{m s}+\pi_{m}^{*}\left(t_{m q} p_{m}^{*} i_{*}\left(\hat{g}_{q}\right)\right) \\
= & \sum_{s=1}^{r}\left(a_{q s} t_{s q} t_{q m} / t_{s m}\right) \bar{\sigma}_{m s} \\
& +\sum_{s=1}^{r}\left\{a_{q s}\left(t_{s m} t_{m q} t_{q s}-t_{m s} t_{s q} t_{q m}\right) /\left(m_{s} t_{s m}\right)+b_{q s} t_{m q}\right\} \bar{\lambda}_{m s} \\
& +\pi_{m}^{*}\left(t_{m q} p_{m}^{*} \imath_{*}\left(\hat{g}_{q}\right)+\sum_{s=1}^{r} a_{q s} j_{s *}\left(\gamma_{m s}^{q}\right)\right) .
\end{aligned}
$$

Similarly,

$$
\begin{aligned}
\Theta \circ \bar{\lambda}_{m q}= & \sum_{h, s=1}^{r} a_{h s} \bar{\sigma}_{h s} \circ \bar{\lambda}_{m q}+\sum_{h, s=1}^{r} b_{h s} \bar{\lambda}_{h s} \circ \bar{\lambda}_{m q}+g \circ \bar{\lambda}_{m q} \\
= & \sum_{s=1}^{r}\left\{\left(a_{q s} m_{q} t_{q s} / m_{s}\right)+b_{q s} m_{q}\right\} \bar{\lambda}_{m s} \\
& +\pi_{m}^{*}\left(m_{q} p_{m}^{*} i_{*}\left(\hat{g}_{q}\right)+\sum_{s=1}^{r} a_{q s} j_{s *}\left(\beta_{m s}^{q}\right)\right) .
\end{aligned}
$$

On the other hand,

$$
\Theta \circ g^{\prime}=\Theta \circ\left(\sum_{m=1}^{r} g_{m}^{\prime \prime}\right)=\sum_{m=1}^{r} \Theta \circ g_{m}^{\prime \prime}
$$

Since $\imath=E i^{\prime}$,

$$
\begin{aligned}
\Theta \circ g_{m}^{\prime \prime} & =\left(\sum_{h, s=1}^{r} a_{h s} \bar{\sigma}_{h s}+\sum_{h, s=1}^{r} b_{h s} \bar{\lambda}_{h s}+g\right) \circ\left(i \circ \hat{g}_{m}^{\prime \prime} \circ p_{m} \circ \pi_{m}\right) \\
& =\left(\sum_{h, s=1}^{r}\left(a_{h s} \bar{\sigma}_{h s} \circ i+b_{h s} \bar{\lambda}_{h s} \circ i\right)+g \circ i\right) \circ\left(\hat{g}_{m}^{\prime \prime} \circ p_{m} \circ \pi_{m}\right) .
\end{aligned}
$$

Furthermore, it follows from $\pi_{h} \circ i=i_{h} \circ \pi_{h}^{\prime}$ and $p_{h} \circ i_{h}=0$ that

and

$$
\begin{aligned}
\bar{\sigma}_{h s} \circ i & =\left(\jmath_{s} \circ \bar{\sigma}_{h s} \circ \pi_{h}\right) \circ 2 \\
& =j_{s} \circ \bar{\sigma}_{h s^{\circ}} i_{h} \circ \pi_{h}^{\prime} \\
& =\jmath_{s} \circ\left(t_{s h} i_{s}\right) \circ \pi_{h}^{\prime}=t_{s h} j_{s * l_{s *}}\left(\pi_{h}^{\prime}\right), \\
\bar{\lambda}_{h s} \circ i & =\left(j_{s} \circ \lambda_{h s} \circ \pi_{h}\right) \circ i \\
& =j_{s} \circ\left(\xi_{s} \circ p_{h}\right) \circ\left(i_{h} \circ \pi_{h}^{\prime}\right)=0,
\end{aligned}
$$




$$
\begin{aligned}
g \circ \imath & =\left(\sum_{h=1}^{r} g_{h}\right) \bullet \imath \\
& =\sum_{h=1}^{r} g_{h^{\circ} \imath \quad\left(\text { by } \imath=E i^{\prime}\right)} \\
& =\sum_{h=1}^{r}\left(\imath \circ \hat{g}_{h} \circ p_{h} \circ \pi_{h} \circ \imath\right) \\
& =\sum_{h=1}^{r}\left(\imath \circ \hat{g}_{h} \circ p_{h} \circ \imath_{h} \circ \pi_{h}^{\prime}\right)=0 .
\end{aligned}
$$

Hence

$$
\begin{aligned}
\Theta \circ g^{\prime} & \left.=\sum_{m=1}^{r}\left(\sum_{h, s=1}^{r} a_{h s} t_{s h}\right]_{s *} l_{s *}\left(\pi_{h}^{\prime}\right)\right) \circ\left(\hat{g}_{m}^{\prime \prime} \circ p_{m} \circ \pi_{m}\right) \\
& =\sum_{m=1}^{r} \pi_{m}^{*}\left(p_{m}^{*}\left(\sum_{h, s=1}^{r} a_{h s} t_{s h} J_{s *} i_{s *}\left(\pi_{h}^{\prime}\right)\right) \circ \hat{g}_{m}^{\prime \prime}\right)
\end{aligned}
$$

Therefore,

$$
\Theta \circ \Theta^{\prime}=\sum_{h, s=1}^{r}\left(A_{h s} \bar{\sigma}_{h s}+B_{h s} \bar{\lambda}_{h s}\right)+\sum_{h=1}^{r} \pi_{h}^{*}\left(\Gamma_{h}\right),
$$

where $A_{h s}, B_{h s}$ and $\Gamma_{h}$ are defined by (4.22) and (4.23).

Q.E.D.

\section{§. A representation by matrices.}

Let $A=\left(\theta_{h s}\right)$ and $B=\left(\theta_{h s}^{\prime}\right)$ be any elements of $\operatorname{Mat}\left(r,\left[K_{h}, K_{s}\right]\right)$, where $\theta_{h s}, \theta_{h s}^{\prime} \in\left[K_{h}, K_{s}\right]$ for $1 \leqq h, s \leqq r$. Then the matrix multiplication

$$
\operatorname{Mat}\left(r,\left[K_{h}, K_{s}\right]\right) \times \operatorname{Mat}\left(r,\left[K_{h}, K_{s}\right]\right) \longrightarrow \operatorname{Mat}\left(r,\left[K_{h}, K_{s}\right]\right)
$$

is defined by

$$
A \cdot B=C=\left(\theta_{h s}^{\prime \prime}\right) \text {, }
$$

where

$$
\theta_{h s}^{\prime \prime}=\sum_{m=1}^{r} \theta_{m s^{\circ}}^{\prime} \theta_{h m} \quad \text { for } 1 \leqq h, s \leqq r .
$$

In general, this matrix-multiplication does not necessarily satisfy the associative law. However, it is easy to see that the associative law holds if $\jmath_{*}:[K, K]$ $\rightarrow$ Mat $\left(r,\left[K_{h}, K_{s}\right]\right)$ is a multicative homomorphism since $j_{*}$ is surjective. Furthermore, the element $E=\left(\delta_{h s} \sigma_{h s}\right)$ is an unit element, where $\delta_{h s}$ denotes the Kronecker delta.

Here we quote

PROPOSITION 5.4 (A.J. Sieradski, [10]). If $n \geqq k+3$, the induced homomorphism 


$$
\jmath *:[K, K] \stackrel{\cong}{\longrightarrow} \operatorname{Mat}\left(r,\left[K_{h}, K_{s}\right]\right)
$$

is an additive and multiplicative isomorphism, where the multiplication of $[K, K]$ is defined by the composition of maps and one of Mat $\left(r,\left[K_{h}, K_{s}\right]\right)$ is given by the matrix-multiplication.

If $n \geqq k+3$, then it follows from the Freudenthal suspension theorem that $\pi_{n+k+1}\left(\stackrel{r}{V}^{n}\right)=E \pi_{n+k}\left(\bigvee^{r} S^{n-1}\right)$ and $[K, K]$ may be considered as a (non-commutative) ring. Then, by using Theorem $C$ and (5.2) we have

Theorem D. Let $n \geqq k+3$, and for each elements $\Theta$ and $\Theta^{\prime} \in[K, K]$ we can put

$$
\Theta=\left(\theta_{h s}\right) \in \operatorname{Mat}\left(r,\left[K_{h}, K_{s}\right]\right)
$$

and

where

$$
\Theta^{\prime}=\left(\theta_{h s}^{\prime}\right) \in \operatorname{Mat}\left(r,\left[K_{h}, K_{s}\right]\right)
$$

$$
\begin{aligned}
& \theta_{h s}=a_{h s} \sigma_{h s}+b_{h s} \lambda_{h s}+g_{h s} \in\left[K_{h}, K_{s}\right], \\
& \theta_{h s}^{\prime}=a_{h s}^{\prime} \sigma_{h s}+b_{h s}^{\prime} \lambda_{h s}+g_{h s}^{\prime} \in\left[K_{h}, K_{s}\right], \\
& g_{h s}=p_{h}^{*} i_{s *}\left(\hat{g}_{h s}\right) \in G_{h s}, \\
& g_{h s}^{\prime}=p_{h}^{*} i_{s *}\left(\hat{g}_{h s}^{\prime}\right) \in G_{h s}, \\
& a_{h s}, a_{h s}^{\prime}, b_{h s}, b_{h s}^{\prime} \in Z \quad \text { for } 1 \leqq h, s \leqq r .
\end{aligned}
$$

Then the multiplicative structure of $[K, K]$ is given by the formula:

$$
\Theta \circ \Theta^{\prime}=\left(\theta_{h s}^{\prime \prime}\right) \in[K, K] \cong \operatorname{Mat}\left(r,\left[K_{h}, K_{s}\right]\right),
$$

where

$$
\theta_{h s}^{\prime \prime}=A_{h s} \sigma_{h s}+B_{h s} \lambda_{h s}+\Gamma_{h s} \in\left[K_{h}, K_{s}\right],
$$

and

$$
\begin{aligned}
\Gamma_{h s}= & \sum_{q=1}^{r}\left\{\left(a_{h q}^{\prime} t_{h q}+b_{h q}^{\prime} m_{q}\right) p_{h}^{*} i_{s *}\left(\hat{g}_{q s}\right)\right. \\
& \left.+a_{q s} t_{s q} p_{h i_{s *}^{*}}\left(\hat{g}_{h q}^{\prime}\right)+b_{h q}^{\prime} a_{q s} \beta_{h s}^{q}+a_{h q}^{\prime} a_{q s} \gamma_{h s}^{q}\right\} \quad \text { for } 1 \leqq h, s \leqq r .
\end{aligned}
$$

Remark 5.11. We suppose that $n \geqq k+3$ and

$$
\alpha_{h}=\alpha_{1} \quad \text { for } 1 \leqq h \leqq r .
$$


Then it is easy to see that

and

$$
\begin{array}{ll}
\beta_{h s}^{q}=\gamma_{h s}^{q}=0 & \text { for } 1 \leqq h, s, q \leqq r, \\
t_{h s}=1 & \text { for } 1 \leqq h, s \leqq r,
\end{array}
$$

$$
m_{h}=m_{1} \quad \text { for } 1 \leqq h \leqq r .
$$

Under the above assumption, by using Corollary B we have

$$
[K, K] \cong \operatorname{Mat}(r, Z) \oplus \operatorname{Mat}(r, Z) \oplus \operatorname{Mat}\left(r, G_{1}\right)
$$

as an additive group.

where

$$
\begin{aligned}
G_{1} & =\pi_{n+k+1}\left(S^{n}\right) / \alpha_{1 * \pi_{n+k+1}\left(S^{n+k}\right)} \\
& =\pi_{n+k+1}\left(S^{n}\right) / E \alpha_{1}^{*} \pi_{n+1}\left(S^{n}\right) .
\end{aligned}
$$

Then, by using Theorem $\mathrm{D}$, the multiplication is given by

$$
\begin{aligned}
\Theta \circ \Theta^{\prime}= & (A, B, \Gamma) \circ\left(A^{\prime}, B^{\prime}, \Gamma^{\prime}\right) \\
= & \left(A^{\prime} A, A^{\prime} B+B^{\prime} A+m_{1} B^{\prime} B,\left(A^{\prime}+m_{1} B^{\prime}\right) \Gamma+\Gamma^{\prime} A\right) \\
& \text { for } \Theta=(A, B, \Gamma) \in[K, K] \text { and } \Theta^{\prime}=\left(A^{\prime}, B^{\prime}, \Gamma^{\prime}\right) \in[K, K] .
\end{aligned}
$$

Here we note the formula

$$
\begin{aligned}
& \left(\begin{array}{ccc}
A^{\prime} & 0 & 0 \\
0 & A^{\prime}+m_{1} B^{\prime} & \Gamma^{\prime} \\
0 & 0 & A^{\prime}
\end{array}\right)\left(\begin{array}{ccc}
A & 0 & 0 \\
0 & A+m_{1} B & \Gamma \\
0 & 0 & A
\end{array}\right) \\
& =\left(\begin{array}{ccc}
A^{\prime} A & 0 & 0 \\
0 & A^{\prime} A+m_{1}\left(A^{\prime} B+B^{\prime} A+m_{1} B^{\prime} B\right) & \left(A^{\prime}+m_{1} B^{\prime}\right) \Gamma+\Gamma^{\prime} A \\
0 & 0 & A^{\prime} A
\end{array}\right) .
\end{aligned}
$$

For each integer $m$ and an abelian group $H$, let $M(r ; m, H)$ denote the ring of matrices of the form

$$
\left(\begin{array}{ccc}
A & 0 & 0 \\
0 & A+m B & \Gamma \\
0 & 0 & A
\end{array}\right) \quad \begin{gathered}
\text { for } A, B \in \operatorname{Mat}(r, Z), \text { and } \\
\Gamma \in \operatorname{Mat}(r, H) .
\end{gathered}
$$

Hence it follows from (5.14), (5.15) and (5.16) that we obtain

THEOREM E. Let $n \geqq k+3$, and we suppose that 
Then

$$
\alpha_{h}=\alpha_{1} \quad \text { for } 1 \leqq h \leqq r .
$$

where

$$
[K, K] \cong M\left(r ; m_{1}, G_{1}\right) \quad \text { as a ring, }
$$

the integer $m_{1}$ is the order of $\alpha_{1}$ and

$$
G_{1}=\pi_{n+k+1}\left(S^{n}\right) / \alpha_{1 * \pi} \pi_{n+k+1}\left(S^{n+k}\right)=\pi_{n+k+1}\left(S^{n}\right) / E \alpha_{1}^{*} \pi_{n+1}\left(S^{n}\right) .
$$

We denote by $\operatorname{Inv}(M)$ the group of all multiplicative invertible elements of a ring $M$.

Then we also have

COROLLARY F. Under the same assumptions as above, we have an isomorphism

$$
\operatorname{Eq}(K) \cong \operatorname{Inv}\left(M\left(r ; m_{1}, G_{1}\right)\right) \text {. }
$$

EXAMPLE 5.17. Let $n \geqq m+4$. Then

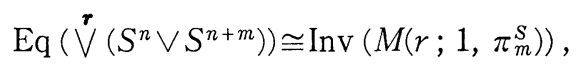

where $\pi_{m}^{S}$ is the $m$-th stable homotopy group of spheres.

EXAMPLE 5.18. Let $m \geqq 2$ and $C P^{2}$ be the two dimensional complex projective space.

Then

$$
\mathrm{Eq}\left(\stackrel{r}{V}^{m} C P^{2}\right) \cong \operatorname{Inv}(M(r ; 2,0)) .
$$

Proof. Let $\eta_{2} \in \pi_{3}\left(S^{2}\right)$ be the Hopf map and we put $\eta_{t}=E^{t-2} \eta_{2}$ for $t \geqq 2$. Then it is well-known that $E^{m} C P^{2}=S^{2+m} \bigcup_{\eta_{2+m}} e^{4+m}$ and $\pi_{t+2}\left(S^{t}\right)=\left\{\eta_{t^{\circ}} \eta_{t+1}\right\} \cong Z / 2 Z$ $=Z_{2}$ for $t \geqq 2$. Thus the assertion easily follows from Corollary F. Q.E.D.

A similar argument also shows

EXAMPLE 5.19. Let $m \geqq 4$ and $H P^{2}$ be the two dimensional quaternion projective space.

Then

$$
\mathrm{Eq}\left(\bigvee^{r} E^{m} H P^{2}\right) \cong \operatorname{Inv}(M(r ; 24,0))
$$

\section{$\S 6$. The applications.}

In this section, we will investigate the action of $\mathrm{Eq}(K)$ on the homotopy group $\pi_{2 n+k}(K)$

$$
\mathrm{Eq}(K) \times \pi_{2 n+k}(K) \longrightarrow \pi_{2 n+k}(K)
$$


which is defined by the composition of maps, and give the another proof of Ishimoto's main results given in [3], which classify the connected sum of sphere bundles over a sphere with cross-sections up to homotopy.

First, we recall several basic results about sphere bundles over a sphere. (See in detail [4]) Let $X$ be a $n$-sphere bundle over $(n+k+1)$-sphere which admits a cross-section, and consider the part of the homotopy exact sequence of the fibration $S O(n) \rightarrow S O(n+1) \rightarrow S O(n+1) / S O(n) \cong S^{n}$

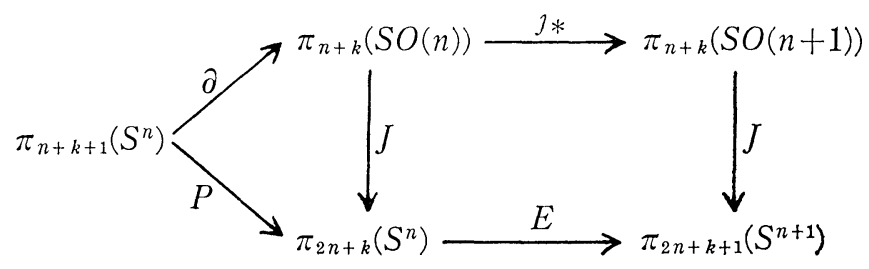

Fig. (6.2)

where $J$ means the classical $J$-homomorphism and the homomorphism $P$ is defined by Whitehead product, i. e.

$$
P(\zeta)=\left[\zeta, \iota_{n}\right] \quad \text { for } \zeta \in \pi_{n+k+1}\left(S^{n}\right) .
$$

Furthermore, it is well-known that

$$
P=-J \circ \partial \text { and } E \circ J=-J \circ J * .
$$

We denote by $\chi(X)$ the characteristic element of $X$. Since $X$ has a cross-section, there exists some element $\gamma \in \pi_{n+k}(S O(n))$ satisfying

$$
\chi(X)=\jmath *(\gamma) .
$$

Then it follows from (6.2) that the element $J(\gamma) \in J \pi_{n+k}(S O(n))$ is uniquely determined up to $P \pi_{n+k+1}\left(S^{n}\right)$, and so we define the invariant $\lambda(X)$ by

$$
\lambda(X)=\{J(\gamma)\} \in J \pi_{n+k}(S O(n)) / P \pi_{n+k+1}\left(S^{n}\right) .
$$

By using this invariant, I.M. James and J.H.C. Whitehead classified the homotopy type of sphere bundles over a sphere with cross-sections as follows:

Theorem 6.7 (I.M. James and J.H.C. Whitehead, [4]). Let $X_{1}$ and $X_{2}$ be $n$-sphere bundles over the $(n+k+1)$-sphere which admit cross-sections, and $n \geqq k+3$.

Then $X_{1}$ and $X_{2}$ are of the same homotopy type if and only if $\lambda\left(X_{1}\right)= \pm \lambda\left(X_{2}\right)$.

Let $X_{h}$ be a $n$-sphere bundle over a $(n+k+1)$-sphere for $1 \leqq h \leqq r$. We denote $\#_{h=1}^{r} X_{h}$ the connected sum of the total spaces $X_{h}, h=1,2, \cdots, r$. Let $X_{s}^{\prime}$, $s=1,2, \cdots, r^{\prime}$, be another set of such bundles. Then it is easy to see that, if $\# h=1 X_{h}$ and $\#_{s=1}^{r^{\prime}} X_{s}^{\prime}$ are of the same homotopy type, $r^{\prime}$ must be equal to $r$ by the homological reason. 
Then the aim of this section is to extend the above result to the case of connected sums of sphere bundles over a sphere which admit cross-sections, and our result is stated as follows:

Theorem G (H. Ishimoto, [3]). Let $n \geqq k+3$ and $k \geqq 1$. Let $X_{h}$ and $X_{h}^{\prime}$ be $n$-sphere bundles over $(n+k+1)$-spheres which admit cross-sections, for $1 \leqq h \leqq r$.

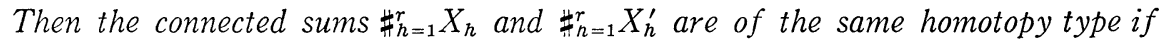
and only of there exists an unimodular $(r \times r)$-matrix $A \in \mathrm{GL}(r, Z)$ such that

$$
\left(\begin{array}{c}
\lambda\left(X_{1}^{\prime}\right) \\
\vdots \\
\vdots \\
\lambda\left(X_{r}^{\prime}\right)
\end{array}\right)=A \cdot\left(\begin{array}{c}
\lambda\left(X_{1}\right) \\
\vdots \\
\vdots \\
\lambda\left(X_{r}\right)
\end{array}\right)
$$

where the abelian group $J \pi_{n+k}(S O(n)) / P \pi_{n+k+1}\left(S^{n}\right)$ is considered as a left $Z$-module.

Remark 6.9. We suppose that the attaching class $\alpha_{h}=0$. Then $m_{h}=1$ and $K_{h}=S^{n} \vee S^{n+k+1}$.

Furthermore, we may assume

(6.10) the map $p_{h}$ is a retraction map to the second factor

and that

$$
p_{h}: S^{n} \vee S^{n+k+1} \longrightarrow S^{n},
$$

(6.11) the map $\xi_{h}$ is an inclusion map

$$
\xi_{h}: S^{n+k+1} \longrightarrow S^{n} \vee S^{n+k+1} .
$$

Proof of Theorem G. We put

$$
\lambda\left(X_{h}\right)=\left\{J\left(\gamma_{h}\right)\right\} \quad \text { and } \quad \lambda\left(X_{h}^{\prime}\right)=\left\{J\left(\gamma_{h}^{\prime}\right)\right\} \quad \text { for } 1 \leqq h \leqq r,
$$

where $\gamma_{h}, \gamma_{h}^{\prime} \in \pi_{n+k}(S O(n))$. It is well-known that each complex $X_{h}$ (resp. $X_{h}^{\prime}$ ) has the cell-decomposition

$$
X_{h}=K_{h} \bigcup_{\rho_{h}} e^{2 n+k+1},\left(\text { resp. } X_{h}^{\prime}=K_{h} \bigcup_{\rho_{h}^{\prime}} e^{2 n+k+1}\right),
$$

where

$$
\begin{aligned}
K_{h} & =S^{n} \vee S^{n+k+1}, \quad \text { and } \\
\rho_{h} & =i_{h *} J\left(\gamma_{h}\right)+\left[\xi_{h}, i_{h}\right] \in \pi_{2 n+k}\left(K_{h}\right) . \\
\text { (resp. } \quad \rho_{h}^{\prime} & \left.=i_{*} J\left(\gamma_{h}^{\prime}\right)+\left[\xi_{h}, i_{h}\right] \in \pi_{2 n+k}\left(K_{h}\right) .\right)
\end{aligned}
$$

Therefore, $\sharp_{h=1}^{r} X_{h}$ and $\sharp_{h=1}^{r} X_{h}^{\prime}$ have the cell-decompositions 


$$
X=\dddot{\sharp}_{h=1}^{r} X_{h}=\left(\bigvee_{h=1}^{r} K_{h}\right) \bigcup_{\rho} e^{2 n+k+1}=K \bigcup_{\rho} e^{2 n+k+1}
$$

and

$$
\begin{aligned}
& X^{\prime}=\prod_{h=1}^{r} X_{h}^{\prime}=\left(\bigvee_{h=1}^{r} K_{h}^{\prime}\right) \bigcup_{\rho^{\prime}} e^{2 n+k+1}=K \bigcup_{\rho^{\prime}} e^{2 n+k+1}, \\
& K=\bigvee_{h=1}^{r} K_{h}=V^{r}\left(S^{n} \bigvee S^{n+k+1}\right),
\end{aligned}
$$

where

$$
\begin{aligned}
& \rho=\sum_{n=1}^{r} j_{h *}\left(\rho_{h}\right) \in \pi_{2 n+k}(K) \quad \text { and } \\
& \rho^{\prime}=\sum_{h=1}^{r} j_{h *}\left(\rho_{h}^{\prime}\right) \in \pi_{2 n+k}(K) .
\end{aligned}
$$

Since $X$ and $X^{\prime}$ are of the same homotopy type it and only if there exists a homotopy equivalence $\Theta \in \mathrm{Eq}(K)$ satisfying $\Theta \circ \rho= \pm \rho^{\prime}$, for our purpose, it is sufficient to investigate the action (6.1).

Let $\Theta=\left(\theta_{h s}\right) \in[K, K]$, where $\theta_{h s}=a_{h s} \sigma_{h s}+b_{h s} \lambda_{h s}+g_{h s}$,

$$
a_{h s}, b_{h s} \in Z \text {, and } g_{h s}=p_{h}^{*} i_{s *}\left(g_{h s}^{\prime}\right) \in G_{h s} \quad \text { for } 1 \leqq h, s \leqq r .
$$

Then we have

$$
\Theta \circ \rho=\sum_{s=1}^{r} \jmath_{s *}\left(\sum_{h=1}^{r} \theta_{h s^{\circ}} \rho_{h}\right)
$$

Here,

$$
\begin{aligned}
& \theta_{h s^{\circ}} \rho_{h}=\theta_{h s^{\circ}} i_{h} \circ J\left(\gamma_{h}\right)+\theta_{h s^{\circ}}\left[\xi_{h}, \imath_{h}\right] \\
& =\left(\theta_{h s} \circ i_{h}\right) \circ J\left(\gamma_{h}\right)+\left[\theta_{h s} \circ \xi_{h}, \theta_{h s} \circ i_{h}\right] .
\end{aligned}
$$

Since $i_{h}=E i_{h}^{\prime}$ and $\sigma_{h s}=i d_{h}=i d_{s}$, it holds that

$$
\begin{aligned}
\theta_{h s^{\circ}} i_{h} & =a_{h s} i_{s}+b_{h s} \lambda_{h s} \circ i_{h}+g_{h s} \circ i_{h} \\
& =a_{h s} i_{s}+b_{h s}\left(\xi_{s} \circ p_{h}\right) \circ i_{h}+i_{s} \circ g_{h s}^{\prime} \circ p_{h} \circ i_{h} \\
& =a_{h s} i_{s} . \quad\left(\text { by } p_{h} \circ i_{h}=0\right)
\end{aligned}
$$

Similarly, it follows from $\xi_{h}=E \xi_{h}^{\prime}$ and $p_{h} \circ \xi_{h}=\ell_{n+k+1}$ that

Hence we have

$$
\begin{aligned}
\theta_{h s^{\circ} \xi_{h}} & =a_{h s} \xi_{s}+b_{h s}\left(\xi_{s} \circ p_{h}\right) \circ \xi_{h}+i_{s} \circ g_{h s}^{\prime} \circ p_{h} \circ \xi_{h} \\
& =\left(a_{h s}+b_{h s}\right) \xi_{s}+i_{s *}\left(g_{h s}^{\prime}\right) .
\end{aligned}
$$

$$
\theta_{h s^{\circ}} \rho_{h}=a_{h s} i_{s} \circ J\left(\gamma_{h}\right)+a_{h s}\left(a_{h s}+b_{h s}\right)\left[\xi_{s}, i_{s}\right]+i_{s *}\left(\left[g_{h s}^{\prime}, \iota_{n}\right]\right) .
$$

First, we suppose that $X$ and $X^{\prime}$ are of the same homotopy type. Then 
there exists a homotopy equivalence $\Theta=\left(\theta_{h s}\right) \in \mathrm{Eq}(K)$ satisfying $\Theta \circ \rho= \pm \rho^{\prime}$. Hence it follows from (6.14) and (6.15) that we have

$$
J\left(\gamma_{s}^{\prime}\right)= \pm \sum_{h=1}^{r} a_{h s} J\left(\gamma_{h}\right) \quad \bmod P \pi_{n+k+1}\left(S^{n}\right),
$$

and

$$
\sum_{h=1}^{r} a_{h s}\left(a_{h s}+b_{h s}\right)= \pm 1 \quad \text { for } 1 \leqq s \leqq r .
$$

On the other hand, since $\Theta=\left(\theta_{h s}\right) \in \mathrm{Eq}(K)$, by using (5.17) we have $A=\left( \pm a_{h s}\right) \in$ $\mathrm{GL}(r, Z)$. Hence the unimodular matrix A satisfies the condition (6.8).

Conversely, we suppose that there exists some unimodular matrix $A=\left(a_{h s}\right)$ $\in \mathrm{GL}(r, Z)$ satisfying the condition (6.8). However, considering (6.7), without loss of generality we may assume

$$
J\left(\gamma_{s}^{\prime}\right)=\sum_{h=1}^{r} a_{h s} J\left(\gamma_{h}\right) \quad \text { for } 1 \leqq s \leqq r .
$$

We put $\Theta=\left(\theta_{h s}\right)=\left(a_{h s} \sigma_{h s}+b_{h s} \lambda_{h s}\right) \in[K, K]$, and $B=\left(b_{h s}\right) \in \operatorname{Mat}(r, Z)$. Then it follows from (5.17) that

$$
\Theta \in \mathrm{Eq}(K) \quad \text { if and only if } A+B \in \mathrm{GL}(r, Z) .
$$

On the other hand, by using (6.15) and (6.17), we have

$$
\begin{aligned}
\sum_{h=1}^{r} \theta_{h s} \circ \rho_{h} & =\sum_{h=1}^{r} a_{h s} i_{s *} J\left(\gamma_{h}\right)+\sum_{h=1}^{r} a_{h s}\left(a_{h s}+b_{h s}\right)\left[\xi_{s}, i_{s}\right] \\
& =\imath_{s *} J\left(\gamma_{s}^{\prime}\right)+\sum_{h=1}^{r} a_{h s}\left(a_{h s}+b_{h s}\right)\left[\xi_{s}, \imath_{s}\right] .
\end{aligned}
$$

Hence it follows from (6.13), (6.14) and (6.15) that

$$
\Theta \circ \rho=\rho^{\prime} \quad \text { if and only if } \sum_{h=1}^{r} a_{h s}\left(a_{h s}+b_{h s}\right)=1 \text { for } 1 \leqq s \leqq r .
$$

Now we put $B=\left({ }^{t} A\right)^{-1}-A \in \operatorname{Mat}(r, Z)$. Then we also obtain ${ }^{t} A(A+B)=E_{r}$, where $E_{r}$ is the identity matrix of degree $r$. Hence, by using (6.18) and (6.19), we have

$$
\Theta \in \operatorname{Eq}(K) \text { and } \Theta \circ \rho=\rho^{\prime} .
$$

Therefore $X$ and $X^{\prime}$ are of the same homotopy type. This completes the proof.

Q.E.D.

\section{REFERENCES}

[1] Y. Ando And K. Yamaguchi, On the homotopy self-equivalences of the product $A \times B$, Proc. Japan Acad., 58 (1982), 323-325.

[2] W. Barcus AND M.G. Barratt, On the homotopy classification of a fixed map, 
Trans. Amer. Math. Soc., 88 (1958), 57-74.

[3] H. Isнimoto, Homotopy classification of connected sums of sphere bundles over spheres I, Nagoya Math. J., 88 (1981), 15-36.

[4] I. M. JAmes AND J.H.C. WhiteheAd, The homotopy theory of sphere bundles over spheres I, Proc. London Math. Soc., 4 (1954), 196-218.

[5] P. J. KAHN, Self-equivalences of (n-1)-connected 2n-manifolds, Math. Ann., 180 (1969), 26-47.

[6] S. OKA, Groups of self-equivalences of certain complexes, Hiroshima Math. J., 2 (1972), 285-298.

[7] S. OKa, N. Sawashita And M. Sugawara, On the group of selfequivalences of a mapping cone, Hiroshima Math. J., 4 (1974), 9-28.

[8] S. SASAO, Homotopy types of spherical fibre spaces over spheres, Pacific J. Math., 52 (1974), 207-219.

[9] S. SASAO AND Y. Ando, On the group $\varepsilon(K(\pi, 1) \times X)$ for 1 -connected $C W$-complexes $X$, Kodai Math. J., 5 (1982), 65-70.

[10] A. J. Sieradski, Twisted self-homotopy equivalences, Pacific. J. Math. 34 (1970), 789-802.

[11] I. TAmura, On the classification of sufficiently connected manifolds, J. Math. Soc. Japan, 20 (1968), 371-389.

[12] H. TodA, Composition methods in homotopy groups of spheres, Annals of Math. Studies. 49 (1962), Priceton Univ. Press.

[13] C.T.C. WALL, Classification of (n-1)-connected 2n-manifolds, Annals of Math., 75 (1962), 163-189.

[14] K. Yamaguchi, On the homotopy type of $C W$-complexes with the form $S^{2} \cup e^{4} \cup e^{6}$, Kodai Math. J., 5 (1982), 303-312.

Department of Mathematics

TOKyo Institute of TECHNOLOGY

Oh-OKayama, Meguro, Tokyo

JAPAN 